\title{
COLDz: Shape of the CO Luminosity Function at High Redshift and the Cold Gas History of the Universe
}

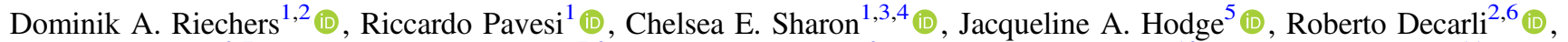 \\ Fabian Walter ${ }^{2,7}$ (10), Christopher L. Carilli ${ }^{7,8}$ (1) , Manuel Aravena ${ }^{9}$ (1), Elisabete da Cunha ${ }^{10}$ (10), Emanuele Daddi ${ }^{11}$ (1),

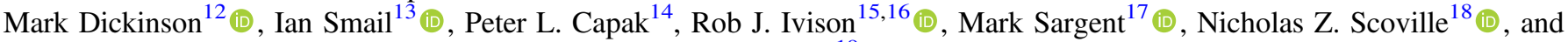 \\ Jeff Wagg ${ }^{19}$ \\ ${ }^{1}$ Department of Astronomy, Cornell University, Space Sciences Building, Ithaca, NY 14853, USA; riechers@ @ cornell.edu \\ ${ }_{2}$ Max-Planck-Institut für Astronomie, Königstuhl 17, D-69117 Heidelberg, Germany \\ ${ }^{3}$ Department of Physics \& Astronomy, McMaster University, 1280 Main Street West, Hamilton, ON L85-4M1, Canada \\ ${ }^{4}$ Yale-NUS College, \#01-220, 16 College Avenue West, 138527, Singapore \\ ${ }^{5}$ Leiden Observatory, Leiden University, P.O. Box 9513, 2300 RA Leiden, The Netherlands \\ ${ }^{6}$ INAF-Osservatorio di Astrofisica e Scienza dello Spazio, via Gobetti 93/3, I-40129, Bologna, Italy \\ ${ }^{7}$ National Radio Astronomy Observatory, Pete V. Domenici Array Science Center, P.O. Box O, Socorro, NM 87801, USA \\ ${ }^{8}$ Cavendish Astrophysics Group, University of Cambridge, Cambridge, CB3 OHE, UK \\ ${ }^{9}$ Núcleo de Astronomía, Facultad de Ingeniería y Ciencias, Universidad Diego Portales, Av. Ejército 441, Santiago, Chile \\ ${ }^{10}$ Research School of Astronomy and Astrophysics, Australian National University, Canberra, ACT 2611, Australia \\ ${ }^{11}$ Laboratoire AIM, CEA/DSM-CNRS-Universite Paris Diderot, Irfu/Service d'Astrophysique, CEA Saclay, Orme des Merisiers, F-91191 Gif-sur-Yvette cedex, \\ France \\ ${ }^{12}$ National Optical Astronomy Observatory, 950 N. Cherry Avenue, Tucson, AZ 85719, USA \\ ${ }^{13}$ Centre for Extragalactic Astronomy, Department of Physics, Durham University, South Road, Durham DH1 3LE, UK \\ ${ }^{14}$ Spitzer Science Center, California Institute of Technology, MC 220-6, 1200 East California Boulevard, Pasadena, CA 91125, USA \\ ${ }^{15}$ European Southern Observatory, Karl-Schwarzschild-Straße 2, D-85748 Garching, Germany \\ ${ }^{16}$ Institute for Astronomy, University of Edinburgh, Royal Observatory, Blackford Hill, Edinburgh EH9 3HJ, UK \\ ${ }^{17}$ Astronomy Centre, Department of Physics and Astronomy, University of Sussex, Brighton, BN1 9QH, UK \\ 18 Astronomy Department, California Institute of Technology, MC 249-17, 1200 East California Boulevard, Pasadena, CA 91125, USA \\ ${ }^{19}$ SKA Organization, Lower Withington, Macclesfield, Cheshire SK11 9DL, UK \\ Received 2018 August 10; revised 2018 November 27; accepted 2019 January 4; published 2019 February 6
}

\begin{abstract}
We report the first detailed measurement of the shape of the CO luminosity function at high redshift, based on $>320 \mathrm{hr}$ of the NSF's Karl G. Jansky Very Large Array (VLA) observations over an area of $\sim 60 \operatorname{arcmin}^{2}$ taken as part of the CO Luminosity Density at High Redshift (COLDz) survey. COLDz "blindly" selects galaxies based on their cold gas content through $\mathrm{CO}(J=1 \rightarrow 0)$ emission at $z \sim 2-3$ and $\mathrm{CO}(J=2 \rightarrow 1)$ at $z \sim 5-7$ down to a $\mathrm{CO}$ luminosity limit of $\log \left(L_{\mathrm{CO}}^{\prime} / \mathrm{K} \mathrm{km} \mathrm{s}^{-1} \mathrm{pc}^{2}\right) \simeq 9.5$. We find that the characteristic luminosity and bright end of the CO luminosity function are substantially higher than predicted by semi-analytical models, but consistent with empirical estimates based on the infrared luminosity function at $z \sim 2$. We also present the currently most reliable measurement of the cosmic density of cold gas in galaxies at early epochs, i.e., the cold gas history of the universe, as determined over a large cosmic volume of $\sim 375,000 \mathrm{Mpc}^{3}$. Our measurements are in agreement with an increase of the cold gas density from $z \sim 0$ to $z \sim 2-3$, followed by a possible decline toward $z \sim 5-7$. These findings are consistent with recent surveys based on higher- $J$ CO line measurements, upon which $\mathrm{COLDz}$ improves in terms of statistical uncertainties by probing $\sim 50-100$ times larger areas and in the reliability of total gas mass estimates by probing the low- $J$ CO lines accessible to the VLA. Our results thus appear to suggest that the cosmic star formation rate density follows an increased cold molecular gas content in galaxies toward its peak about 10 billion years ago, and that its decline toward the earliest epochs is likely related to a lower overall amount of cold molecular gas (as traced by $\mathrm{CO}$ ) bound in galaxies toward the first billion years after the Big Bang.
\end{abstract}

Key words: cosmology: observations - galaxies: active - galaxies: formation - galaxies: high-redshift - galaxies: starburst - radio lines: galaxies

\section{Introduction}

Our basic understanding of galaxy evolution and the buildup of stellar mass in galaxies throughout the history of the universe is founded on detailed measurements of the star formation rate density ${ }^{20}$ as a function of cosmic time (or redshift), the "star formation history of the universe," and measurements of the stellar mass density in galaxies at different cosmic epochs (see Madau \& Dickinson 2014 for a review). Indepth studies of significant samples of high-redshift galaxies

\footnotetext{
${ }^{20}$ Throughout this work, densities in star formation rate, stellar mass, or gas mass refer to cosmic densities (i.e., the amount of material in galaxies per unit co-moving cosmic volume) unless stated otherwise.
}

appear to indicate that the changes in this growth history at different epochs are largely driven by the cold molecular gas properties of galaxies (e.g., Daddi et al. 2010; Tacconi et al. 2013, 2018; Genzel et al. 2015; Scoville et al. 2016) and by the growth rate of dark matter halos (e.g., Bouché et al. 2010; Genel et al. 2010; Faucher-Giguère et al. 2011). The cold gas constitutes the fuel for star formation (see Carilli \& Walter 2013 for a review), such that a higher gas content (e.g., driven by high gas accretion rates) or a higher efficiency of converting gas into stars (e.g., driven by galaxy mergers, or by ubiquitous shocks due to high gas flow rates) can lead to increased star formation activity, and thus to a more rapid growth of galaxies (e.g., Davé et al. 2012). 
To better understand how the gas supply in galaxies moderates the star formation rate density in galaxies at early epochs, it is desirable to complement targeted studies with an integrated measurement of the cold molecular gas density in galaxies at the same epochs; i.e., the "cold gas history of the universe." Surveys of cold gas ideally target rotational lines of $\mathrm{CO}$, which is the most common tracer of the molecular gas mass in galaxies, to measure the CO luminosity function at different cosmic epochs in a "molecular deep field" study. The distribution mean of the $\mathrm{CO}$ luminosity function then provides a reliable measurement of the cold molecular gas density at a given redshift (Carilli \& Walter 2013; see, e.g., Scoville et al. 2017 for an alternative approach). The first of these efforts have recently been carried out in the Hubble Deep Field North (HDF-N) and the Hubble Ultra Deep Field (H-UDF) with the IRAM Plateau de Bure Interferometer (PdBI) and the Atacama Large Millimeter/ submillimeter Array (ALMA; the ASPECS-Pilot program) at $3 \mathrm{~mm}$ and $1 \mathrm{~mm}$ wavelengths, covering fields $\sim 0.5$ and $\sim 1 \operatorname{arcmin}^{2}$ in size, respectively (see Decarli et al. 2014; Walter et al. 2016, and references therein). At $z \sim 2-3$, near the peak of the cosmic star formation rate density $\sim 10$ billion years ago, these studies cover $\mathrm{CO}(J=3 \rightarrow 2)$ and higher- $J$ lines. At $z=5-7$, i.e., in the first billion years after the Big Bang, these surveys cover $\mathrm{CO}(J=5 \rightarrow 4)$ and higher- $J$ lines.

The most faithful tracer of total cold gas mass are low- $J \mathrm{CO}$ lines, particularly $\mathrm{CO}(J=1 \rightarrow 0$ ) (see, e.g., Riechers et al. 2006, 2011a, 2011b; Ivison et al. 2011; Aravena et al. 2012, 2014; Bolatto et al. 2015; Daddi et al. 2015; Sharon et al. 2016; Saintonge et al. 2017; Harrington et al. 2018), for which the $\alpha_{\mathrm{CO}}=M_{\mathrm{H}_{2}} / L_{\mathrm{CO}}^{\prime}$ conversion factor from CO luminosity $\left(L_{\mathrm{CO}}^{\prime}\right.$, in units of $\left.\mathrm{K} \mathrm{km} \mathrm{s}^{-1} \mathrm{pc}^{2}\right)$ to $\mathrm{H}_{2}$ gas mass $\left(M_{\mathrm{H}_{2}}\right.$, in units of $\left.M_{\odot}\right)$ has been calibrated locally (see Bolatto et al. 2013 for a review), and for which no assumptions about gas excitation are required to derive the total $\mathrm{CO}$ luminosity. To complement the initial "molecular deep field" studies through improved statistical uncertainties measured over larger cosmic volumes and reduced calibration uncertainties due to gas excitation, we carried out the VLA COLDz survey, ${ }^{21}$ which is "blindly" selecting galaxies through their cold gas content in the $\mathrm{CO}(J=1 \rightarrow 0)$ line at $z \sim 2-3$ and in $\operatorname{CO}(J=2 \rightarrow 1)$ at $z \sim 5-7$, over a $\sim 60 \operatorname{arcmin}^{2}$ region.

The detailed survey parameters, line search and statistical techniques, a catalog of line candidates, and an overview of accompanying papers are presented in the COLDz survey reference paper (Pavesi et al. 2018; hereafter: Paper I). This work focuses on the $\mathrm{CO}$ luminosity function measurements that result from the survey data and also on the implied constraints on the evolution of the cosmic cold gas density in galaxies as a function of redshift.

The rest of this paper is structured as follows. Section 2 provides a brief description of the data. Section 3 summarizes the selection of $\mathrm{CO}$ line candidates and it describes the statistical methods that we used to characterize the survey parameters, before describing the $\mathrm{CO}$ luminosity function and cold gas density measurements. Section 4 provides a discussion of the results in the context of previous surveys and model predictions. Section 5 provides the main conclusions based on our measurements and analysis. We use a concordance, flat $\Lambda \mathrm{CDM}$ cosmology throughout, with $H_{0}=69.6 \mathrm{~km} \mathrm{~s}^{-1} \mathrm{Mpc}^{-1}$, $\Omega_{\mathrm{M}}=0.286$, and $\Omega_{\Lambda}=0.714$ (Bennett et al. 2014).

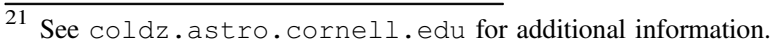

Table 1

Lines, Redshift Ranges, and Volumes Covered by the COLDz Survey (See Figure 1 for Luminosity Limits across the Survey Volume)

\begin{tabular}{lccccc}
\hline \hline Transition & $\begin{array}{c}\nu_{\text {rest }} \\
(\mathrm{GHz})\end{array}$ & $z_{\text {min }}$ & $z_{\max }$ & $\langle z\rangle$ & $\begin{array}{c}\text { Volume } \\
\left(\mathrm{Mpc}^{3}\right)\end{array}$ \\
\hline \multicolumn{7}{c}{ COSMOS “Deep" } & Field $\left(\sim 9 \mathrm{arcmin}^{2} ; \sim 31-39 \mathrm{GHz}\right)$ \\
$\mathrm{CO}(J=1 \rightarrow 0)$ & 115.271 & 1.953 & 2.723 & 2.354 & 20,189 \\
$\mathrm{CO}(J=2 \rightarrow 1)$ & 230.538 & 4.906 & 6.445 & 5.684 & 30,398 \\
\hline \multicolumn{7}{c}{ GOODS-North "Wide" } & Field $(\sim 51$ & $\mathrm{arcmin}^{2} ;$ & $30-38 \mathrm{GHz})$ \\
$\mathrm{CO}(J=1 \rightarrow 0)$ & 115.271 & 2.032 & 2.847 & 2.443 & 131,042 \\
$\mathrm{CO}(J=2 \rightarrow 1)$ & 230.538 & 5.064 & 6.695 & 5.861 & 193,286 \\
\hline
\end{tabular}

Note. The co-moving volume is calculated to the edges of the mosaics, and does not account for varying sensitivity across the mosaics, which is accounted for by the completeness correction.

\section{Data}

The VLA was used to "blindly" observe redshifted $\mathrm{CO}(J=1 \rightarrow 0)$ and $\mathrm{CO}(J=2 \rightarrow 1)$ line emission (rest-frame frequencies: $\nu_{\text {rest }}=115.2712$ and $\left.230.5380 \mathrm{GHz}\right)$ at $z=$ 2.0-2.8 and $z=4.9-6.7$, respectively (VLA program IDs 13A-398 and 14A-214; PI: Riechers), covering a region corresponding to a total co-moving survey volume of $\sim 375,000 \mathrm{Mpc}^{3}$ in both lines combined (see Table 1 for details). Observations covered a 7-pointing mosaic in the COSMOS field (center position: J2000 10:00:20.7; +02:35:17.0), and a 57-pointing mosaic in the GOODS-North field (center position: J2000 12:36:59.0; +62:13:43.5), providing total survey areas of 8.9 and $50.9 \mathrm{arcmin}^{2}$ at 31 and $30 \mathrm{GHz}$, respectively. ${ }^{22}$

Observations in COSMOS and GOODS-North covered the 30.969-39.033 and 29.981-38.001 GHz frequency ranges in a single tuning with $\sim 8 \mathrm{GHz}$ bandwidth (dual polarization) each, respectively, using the Ka band receivers and the 3-bit samplers at a spectral resolution of $2 \mathrm{MHz}\left(17 \mathrm{~km} \mathrm{~s}^{-1}\right.$ at $\left.35 \mathrm{GHz}\right)$. Observations were carried out for a total of $324 \mathrm{hr}$ between 2013 January 26 and 2015 December 18 under good to excellent weather conditions in the $\mathrm{D}$ and $\mathrm{DnC}$ array configurations, and in the $\mathrm{D} \rightarrow \mathrm{DnC}$ and $\mathrm{DnC} \rightarrow \mathrm{C}$ reconfigurations, yielding approximately 93 and $122 \mathrm{hr}$ on source across all configurations and pointings in COSMOS and GOODS-North, respectively. This yields characteristic synthesized beam sizes of $\sim 3^{\prime \prime}$ when imaging the data with natural baseline weighting after data calibration using the CASA package, and approximately 3 times better point-source sensitivity in the smaller COSMOS mosaic. The corresponding $\mathrm{CO}$ luminosity limits across the redshift range are shown in Figure 1. More details on the observations and data reduction are given in Paper I.

\section{Results and Analysis}

\subsection{CO Line Candidates}

Based on our matched filtering algorithm using threedimensional spatial/spectral templates, we find 57 candidate $\mathrm{CO}(J=1 \rightarrow 0)$ and $\mathrm{CO}(J=2 \rightarrow 1)$ line emitters in our survey volume down to signal-to-noise ratio $(\mathrm{S} / \mathrm{N})$ limits of 5.25 and 5.50 in the COSMOS (26 candidates) and GOODS-North (31 candidates) fields, respectively (Paper I). These $\mathrm{S} / \mathrm{N}$ limits are

\footnotetext{
22 The mosaicked images made from individual pointings were trimmed at the outer edges at the $30 \%$ level of the peak sensitivity in each spectral channel.
} 


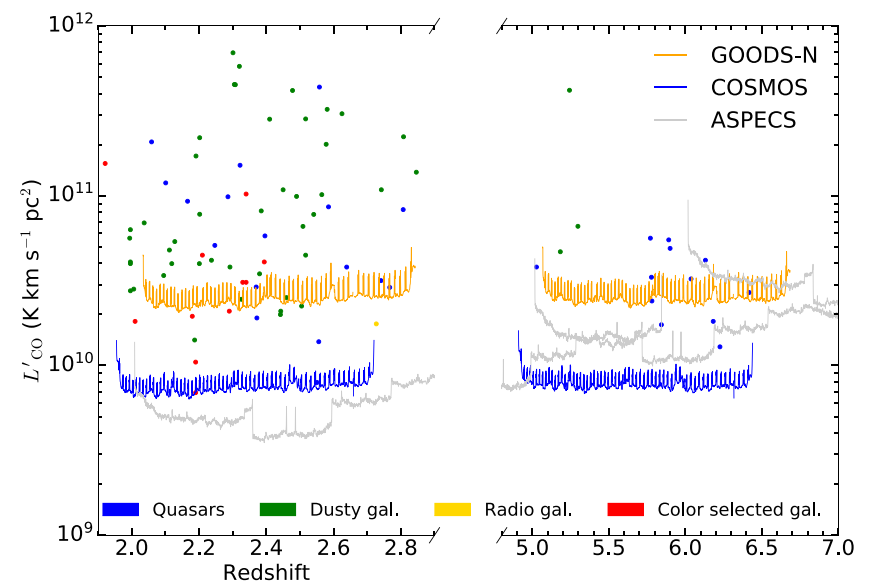

Figure 1. Representative line luminosity detection sensitivity limits as a function of redshift reached by our observations in the COSMOS and GOODSNorth fields, when adopting 5 times the rms noise at a line FHWM of $200 \mathrm{~km} \mathrm{~s}^{-1}$ (see Paper I for variations between individual pointings). The corresponding sensitivity limits of the ALMA ASPECS-Pilot survey in the CO $J=3 \rightarrow 2$ to $7 \rightarrow 6$ lines in the same redshift ranges are shown for comparison (Walter et al. 2016). ASPECS limits are scaled to $\operatorname{CO}(J=1 \rightarrow 0)$ line luminosity limits using the same, representative line excitation correction factors that were adopted by Decarli et al. (2016a) based on Daddi et al. (2015) up to $\mathrm{CO}(J=5 \rightarrow 4)$. For the higher- $J$ lines, we assume an intermediate excitation based on Figure 10 of Daddi et al. (2015), corresponding to brightness temperature ratios of $r_{65} \simeq 0.66$ and $r_{75} \simeq 0.29$ relative to the CO $J=5 \rightarrow 4$ line, respectively. For reference, the colored points show all previous $z>1$ CO detections as compiled by Carilli \& Walter (2013), incorporating updates by Sharon et al. (2016). The colors indicate different galaxy types ("dusty galaxies" includes submillimeter galaxies, extremely red objects, and $24 \mu \mathrm{m}$ selected galaxies, and "color-selected galaxies" includes Lyman-break, BzK, and BMBX galaxies, respectively).

chosen to provide comparable ratios of line candidates with positive fluxes over noise spikes with negative fluxes at matching $\mathrm{S} / \mathrm{N}$ between both fields. This misses at least one independently confirmed $\mathrm{CO}(J=2 \rightarrow 1)$ emitter: $\operatorname{HDF} 850.1$ at $z=5.18$ in the GOODS-North field (Walter et al. 2012), which has a $\mathrm{S} / \mathrm{N}$ of 5.33. Including this source, 7 out of the 58 candidates are presently independently confirmed to be real $\mathrm{CO}(J=1 \rightarrow 0)$ (three sources in COSMOS, one in GOODS-North) ${ }^{23}$ or $\mathrm{CO}(J=$ $2 \rightarrow 1$ ) line emitters (one in COSMOS, two in GOODSNorth) ${ }^{24}$ through the detection of additional CO lines (see, e.g., Paper I; Riechers et al. 2010, 2011a, 2014; Walter et al. 2012). To remain robust against individual, potentially spurious candidates, all of the other line candidates are only used in a probabilistic manner in the statistical analysis of the survey data until independent confirmation is obtained. All of the candidates except the three independently confirmed $\mathrm{CO}$ $(J=2 \rightarrow 1)$ emitters are considered to be $\mathrm{CO}(J=1 \rightarrow 0)$ emitters unless stated otherwise. ${ }^{25}$

\subsection{Statistical Methods}

A detailed description of the statistical properties of the candidate CO line emitter sample is given in Paper I. Here, we briefly summarize the main elements of the methods that are

\footnotetext{
${ }^{23}$ Sources are COLDz.COS.1 to 3 and COLDz.GN.3 (GN19) in Paper I.

24 Sources are COLDz.COS.0 (AzTEC-3), and COLDz.GN.0 (GN10) and 31 (HDF 850.1) in Paper I.

${ }^{25}$ This initial assumption is based on the expectation of a significantly lower space density of bright CO-emitting galaxies at $z \gtrsim 5$ compared to $z<3$ in our current understanding. Alternative scenarios are also discussed below.
}

relevant to the construction of the $\mathrm{CO}$ luminosity function. The main purpose of the statistical analysis is to determine the probability of each line candidate to be real and to determine the completeness of the line search as a function of line luminosity, spatial size, and velocity width. Furthermore, it is necessary to evaluate the probability function of the actual versus measured line luminosity.

\subsubsection{Reliability Analysis: Purity Estimates}

The reliability (or purity/fidelity) of each candidate $\mathrm{CO}$ emitter is given by its probability of corresponding to a real line source. The reliability analysis in this work employs Bayesian techniques that are based on the assumption of symmetry of the noise distribution in the data cubes around zero flux, identifying a real signal as a positive "excess" flux at a given $\mathrm{S} / \mathrm{N}$ evaluated relative to the noise distribution as traced by negative flux features found with the same extraction methods. The posterior probability distributions for the rates of real sources and noise spikes (which provide estimates of the purity) are obtained by modeling the occurence rates of real sources and noise spikes as an inhomogenous Poisson process with different rate models in the $\mathrm{S} / \mathrm{N}$ distribution. Purities are calculated from the posterior probability distributions as a function of the model parameters, which are sampled using a Markov Chain Monte Carlo (MCMC) technique (emcee; Foreman-Mackey et al. 2013). The Poisson rate of the noise distribution is modeled as the tail of a Gaussian in $\mathrm{S} / \mathrm{N}$, based on negative flux features at $S / N \geqslant 4$ in the data. The occurence rate of noise features is then measured by maximizing the likelihood of the noise model, based on all negative features down to the above $\mathrm{S} / \mathrm{N}$ limit. The real source rate is parameterized as a shallow power law, increasing toward lower $\mathrm{S} / \mathrm{N}$ values (based on the conservative assumption that faint sources are more common), normalized at a $\mathrm{S} / \mathrm{N}$ of 6 , with uniform, non-constraining priors on the slope and normalization. Given the simple parameterization as a slowly varying source rate, we include all candidates in the limited $5<\mathrm{S} / \mathrm{N}<9$ range where most candidates are found in the analysis, after confirming that candidates with $\mathrm{S} / \mathrm{N}>9$ (which represent rare sources in our survey) are always assigned a purity of $100 \%$. COLDz.GN.3 (GN19) is the only independently confirmed $\mathrm{CO}(J=1 \rightarrow 0)$ emitter with a $\mathrm{S} / \mathrm{N}<9$, and thus the only secure source considered in the statistical analysis that would be assigned a purity of $<100 \%$ by this method alone. Taking into account the additional information that confirms this source to be real, its purity is known to be $100 \%$ in practice. Thus, the latter, preferred value is adopted in the subsequent analysis. Following this change, all independently confirmed $\mathrm{CO}(J=1 \rightarrow 0)$ emitters have a purity of $100 \% \pm 0 \%$ (see Paper I, Appendix F.1, for further details).

\subsubsection{Artificial Source Analysis: Completeness and Flux Corrections}

The line search and flux extraction methods employed by a "blind" survey, in combination with the observational parameters of the data cubes, determine its completeness. However, the choice of methods may introduce bias into the measurements. A probabilistic analysis of artificial sources of varying fluxes and three-dimensional sizes (i.e., spatial extent and linewidth) injected into the data cubes ${ }^{26}$ and then re-extracted

\footnotetext{
26 The data cubes are used to represent the noise properties because the vast majority of resolution elements are void of signal.
} 
using the same methods as for real candidates is employed here to estimate survey completeness and to account for potential biases. A statistical comparison of injected to extracted artificial source properties is used to correct the $\mathrm{CO}$ luminosity function for the completeness of our line search. This method is also used to correct the measured source fluxes feeding into the luminosity function in a probabilistic manner by estimating biases in the flux extraction procedure ${ }^{27}$ and uncertainties in the flux recovery. Using Bayes theorem, the size, line velocity width, and flux probability distributions found from the artificial source analysis are used in combination with a conservative prior on the fraction of resolved sources to find the probabilistic relationships between measured and real source sizes, line velocity widths, and fluxes. The completeness of the detection process is determined by measuring the fraction of the injected sources that are detected for a given set of source parameters (i.e., line flux, velocity width, and spatial size) as a function of $\mathrm{S} / \mathrm{N}$. This provides an estimate for the fraction of the objects at a given intrinsic line luminosity that are recovered at a given $\mathrm{S} / \mathrm{N}$ threshold, and by accounting for variations of the sensitivity as a function of position and frequency in the mosaics, over what fraction of the survey volume they can be recovered. In the construction of the luminosity function, the probability-weighted completeness is determined by assuming for each luminosity bin that the frequency and line luminosity distributions are uniform within the bin, and no intrinsic spatial size and line velocity width distributions are assumed (i.e., the measured values of the candidates are adopted to determine completeness). To minimize bias, mean values, rather than "per source" completeness values of individual candidates, are adopted for each bin of the luminosity function (see Paper I, Appendix F.3, for further details).

\subsubsection{Construction of the CO Luminosity Function}

The CO luminosity function is assembled by weighting the contribution of each line candidate to a given luminosity bin by its purity and, in a statistical manner (i.e., per luminosity bin, not per source), inversely by its completeness, using the total co-moving cosmic volume covered by the survey (which corresponds to an effective volume $V_{\max }$ for each candidate galaxy due to the completeness corrections and spatial variations of the survey sensitivity). Systematic uncertainties are estimated by calculating the luminosity function with random realizations of the flux and purity estimates (within their statistically well-defined distributions), and with different luminosity bin widths and boundary conditions.

In each luminosity bin, the completeness is determined by averaging over 1000 random realizations (i.e., using a uniform prior) for each combination of spatial size and velocity width covered by the artificial sources, using randomly sampled redshifts to calculate the line fluxes as a basis for the completeness values. We then apply these values as a function of spatial size and velocity width to the probability distribution in these parameters for each candidate, which is effectively using the parameters as weights to the completeness.

Systematic uncertainties are taken into account by calculating 10,000 Monte Carlo realizations of the luminosity function for

\footnotetext{
27 Flux extraction relies on fitting Gaussian line profiles to aperture spectra extracted over a FWHM diameter determined from two-dimensional Gaussian size fitting to velocity-integrated line maps, and thus, is sensitive to the fitted size.
}

every luminosity bin width and center (sampled in intervals of 0.1 dex). We vary the purity values and flux assignments (which are drawn from log-normal distributions for different spatial sizes; see Paper I) for each candidate independently (i.e., allowing them to shift between adjacent luminosity bins) to simulate the uncertainty in their intrinsic fluxes. In the final analysis, luminosity bin widths of 0.5 dex are adopted. A conservative $20 \%$ uncertainty is added to the measured fluxes to account for the uncertainty in the flux calibration of the data. ${ }^{28}$ From the 10,000 realizations, median values and the scatter around the median are calculated for each luminosity bin. The scatter is expressed as the 95th and 5th percentiles for the upper and lower bounds, respectively. Statistical Poisson uncertainties are calculated for each luminosity bin as the relative uncertainty of $1 / \sqrt{N}$, where $N$ corresponds to the number of candidates in the bin (see Paper I, Appendix F.4, for further details).

To further account for systematic uncertainties not fully captured by our statistical treatment, purities are utilized using two different methods. The first method (termed "normal" hereafter; Figure 2, middle panels) draws the purity values for the MCMC sampling used to estimate the allowed range for the luminosity function as random numbers with a normal distribution around the computed values, with a standard deviation of the values themselves, truncated at zero and $100 \%$ purity. This method is motivated by the modest number of line candidates in excess of the tail end of the noise distribution (see Paper I), which limits the precision of more direct methods of measuring the uncertainties. The second method ("uniform"; Figure 2, left-hand panels) more conservatively treats the purities as upper limits, and draws the purity values from a uniform distribution between zero and the computed values. This method is designed to account for the finding that a significant fraction of moderate significance line candidates do not show unambiguous multiwavelength counterparts (see Paper I), which motivates a more conservative treatment of the purity prior. The results of these implementations are consistent within the uncertainties. In the following, we will conservatively adopt a combination of both methods by assuming the outermost upper and lower bounds of the uncertainties between the two methods (Figure 2, right-hand panels).

Given the limited survey statistics due to the moderate number of line candidates, luminosity function constraints are displayed in bins that are not statistically independent throughout but which instead sample the luminosity function in luminosity bins of 0.5 dex width, in steps of 0.1 dex. Independent bins are thus recovered by only considering every fifth bin (see Table 4). Since the candidates are primarily taken into account in a probabilistic manner instead of on a percandidate basis, this choice of partially redundant sampling reveals additional information on the shape of the luminosity function and it shows trends more clearly than broader, more sparsely sampled bins.

\subsection{COLDz CO Luminosity Function}

\subsection{1. $C O(J=1 \rightarrow 0)$ Luminosity Function}

The estimates of the $\operatorname{CO}(J=1 \rightarrow 0)$ luminosity function, which include all candidates except independently confirmed

\footnotetext{
28 This value is higher than the nominal precision of absolute flux calibration at the VLA to account for the fact that some observing runs did not contain one of the "standard" primary flux calibrators (see Paper I for details). This conservative choice only has a minor impact on our results.
} 


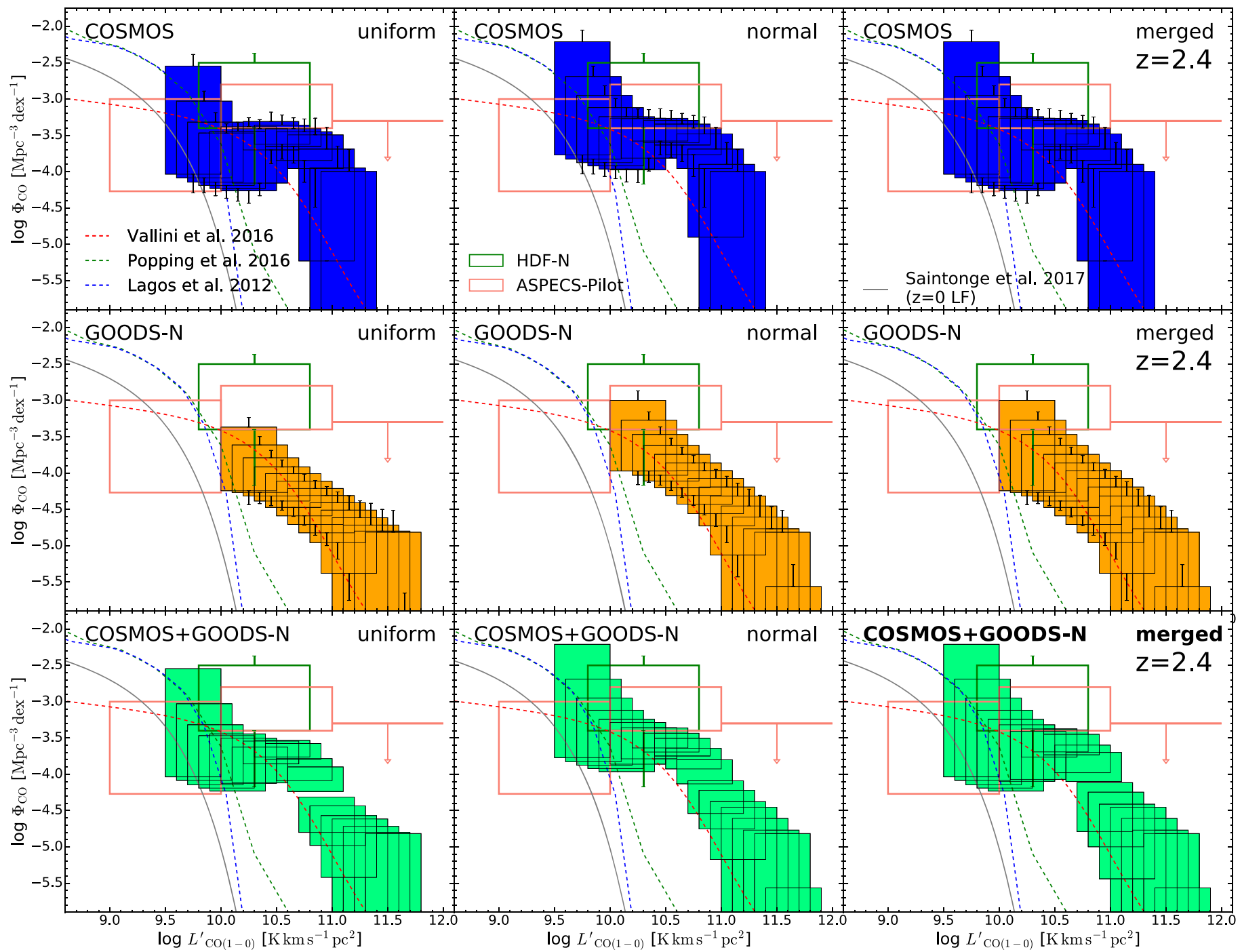

Figure 2. VLA COLDz $\operatorname{CO}(J=1 \rightarrow 0$ ) luminosity function at $\langle z\rangle=2.35$ and 2.44 in the COSMOS (top panels) and GOODS-North (middle panels) fields (shaded boxes), respectively, and the combination of both fields, weighted by the statistical uncertainties in each field (bottom panels), showing the consistency between methods and fields. The left-hand panels show the constraints obtained when conservatively assuming a uniform distribution between zero and the most likely values for the purities. The middle-column panels show the constraints when assuming the most likely values for the purities and assigning $100 \%$ uncertainty to these values, truncated at zero and $100 \%$. The right-hand panels show the composite uncertainties merged from both methods, obtained by assuming the lowest and highest values covered by their respective uncertainty ranges in each bin. Bins have a width of $0.5 \mathrm{dex}$ in $L_{\mathrm{CO}}^{\prime}$, and step through the covered luminosity range in steps of 0.1 dex. As such, individual bins are not statistically independent. Error bars on the boxes indicate Poissonian uncertainties in each bin. Empty green boxes are the constraints on the $\mathrm{CO}(J=3 \rightarrow 2)$ luminosity function at $\langle z\rangle=2.75$ from the PdBI HDF-N survey (Walter et al. 2014). Empty orange boxes are the $\mathrm{CO}(J=3 \rightarrow 2)$ constraints at $\langle z\rangle=2.61$ from the ALMA ASPECS-Pilot survey (Decarli et al. 2016a). A constant $\mathrm{CO}(J=3 \rightarrow 2) / \mathrm{CO}(J=1 \rightarrow 0)$ brightness temperature ratio of $r_{31}=0.42$ has been applied to correct the $\mathrm{CO}(J=3 \rightarrow 2)$ luminosities to $\mathrm{CO}(J=1 \rightarrow 0)$ luminosities for both these surveys. The gray line shows the $z=0$ luminosity function for comparison (updated from Saintonge et al. 2017; A. Saintonge 2018, private communication). The dashed lines are semi-analytical and empirical model predictions (Lagos et al. 2012; Popping et al. 2016; Vallini et al. 2016). All except the COLDz data are the same in all panels.

$\mathrm{CO}(J=2 \rightarrow 1)$ emitters, ${ }^{29}$ are consistent ${ }^{30}$ between both survey fields (Figure 3, left). We thus decided to merge the constraints from both fields through a weighted average in each bin (Figure 2, bottom panel, and Figure 3, middle panel).

\footnotetext{
${ }^{29}$ The level of the CO luminosity function is dominantly determined by independently confirmed sources, such that unconfirmed candidates mainly contribute to the size of the uncertainty ranges. For reference, in the COSMOS field, 1,2 , or 3 secure detections in a bin correspond to $\log \left(\Phi_{\mathrm{CO}} /\right.$ $\left.\mathrm{Mpc}^{-3} \mathrm{dex}^{-1}\right)=-4.00,-3.70$, or -3.53 , respectively. In the GOODS-North field, the same number of detections correspond to values of $-4.82,-4.52$, and -4.34 , respectively.

30 There are some apparent variations between the two fields within the uncertainties, e.g., around the $\log \left(L_{\mathrm{CO}}^{\prime} / \mathrm{K} \mathrm{km} \mathrm{s}^{-1} \mathrm{pc}^{2}\right)=10.5-11.0$ bin, which are likely to be a reflection of cosmic variance.
}

The data reveal the shape of the $\operatorname{CO}(J=1 \rightarrow 0)$ luminosity function at $z \sim 2.4$, which resembles that of a Schechter function. While not a unique solution given current observational constraints, we obtain an estimate of the allowed range of Schechter parameters by fitting the characteristic parameters $L_{\mathrm{CO}}^{\prime *}$ and $\Phi_{\mathrm{CO}}^{*}$ and the power-law slope $\alpha$ to the data (Figure 3, right-hand and Table 2). We adopt the Approximate Bayesian Computation (ABC) method (see, e.g., Cameron \& Pettitt 2012; Weyant et al. 2013; Ishida et al. 2015, and references therein) to derive posterior distributions of the Schechter parameters to account for all selection effects affecting our measurement without having to specify an explicit equivalent likelihood function. 

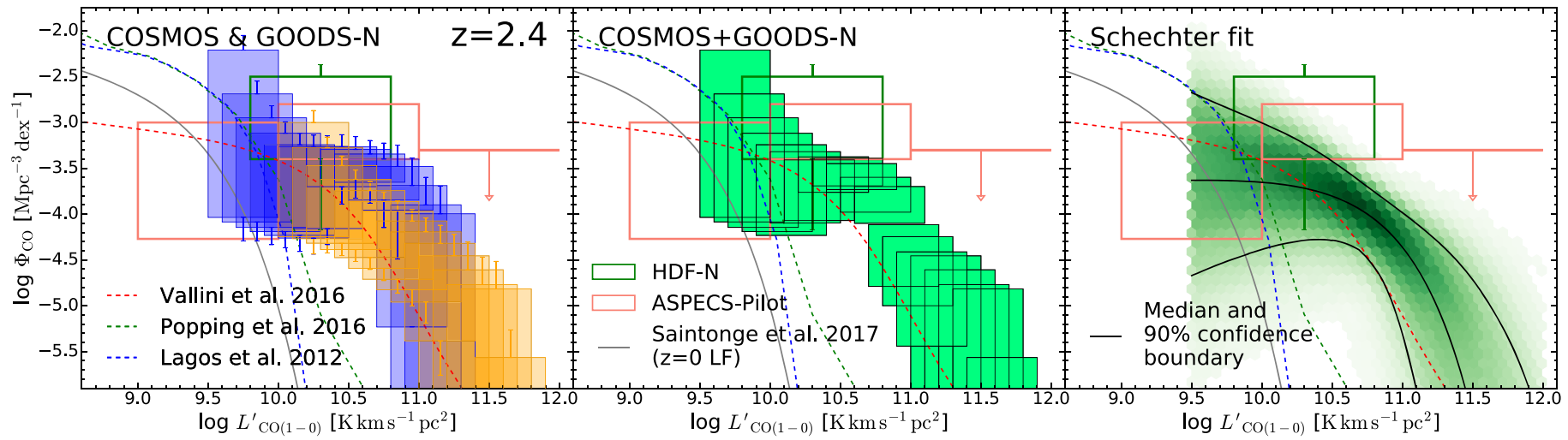

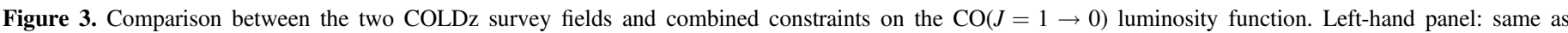

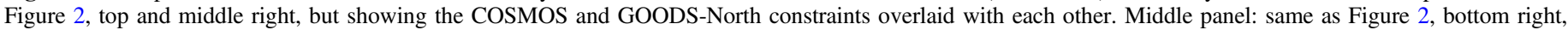

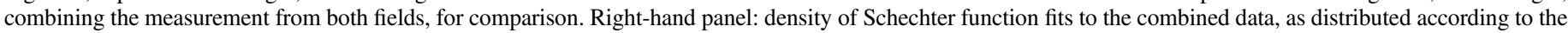

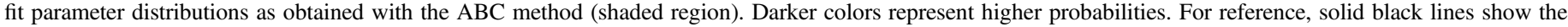

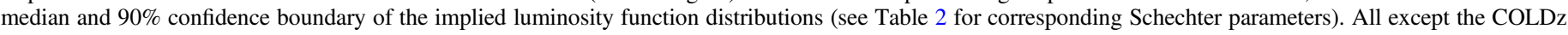
data are the same as in Figure 2 in all panels.

Table 2

Schechter Function Fit Parameter Constraints to the $\mathrm{CO}(J=1 \rightarrow 0)$ Luminosity Function at $z=1.95-2.85$ from COLDz

\begin{tabular}{lccc}
\hline \hline Parameter & 5th Percentile & 50th Percentile & 95th Percentile \\
\hline $\log L_{\text {CO }}^{\prime *}$ & 10.22 & 10.70 & 11.33 \\
$\log \Phi_{\text {CO }}^{*}$ & -4.66 & -3.87 & -3.20 \\
$\alpha$ & -0.78 & 0.08 & 0.99 \\
\hline
\end{tabular}

Note. The CO luminosity function is defined as $\log \Phi_{\mathrm{CO}}=\log \Phi_{\mathrm{CO}}^{*}+$ $\alpha\left(\log L_{\mathrm{CO}}^{\prime}-\log L_{\mathrm{CO}}^{\prime *}\right)-L_{\mathrm{CO}}^{\prime} /\left(L_{\mathrm{CO}}^{\prime *} \ln 10\right)+\log (\ln 10) . L^{\prime}$ is given in units of $\mathrm{K} \mathrm{km} \mathrm{s}^{-1} \mathrm{pc}^{2} . \Phi$ is given in units of $\mathrm{Mpc}^{-3} \mathrm{dex}^{-1}$.

We first assume uniform, unconstraining priors on the Schechter parameters (i.e., $L_{\mathrm{CO}}^{\prime *}, \Phi_{\mathrm{CO}}^{*}$, and $\alpha$ ) to describe the intrinsic distribution of the $\mathrm{CO}$ luminosity density. Following the $\mathrm{ABC}$ method, we then iteratively sample from these priors, which is randomly sampling galaxies with randomly assigned line widths and spatial sizes (according to the same prior distributions as assumed previously) from the assumed distributions. Here, the number of galaxies is assumed to follow a Poisson distribution around the mean. We then correct for completeness and flux recovery in our line search to determine a mock observed sample of galaxies. Next, we compare this mock sample to observations by only including line candidates with a probability equal to their purity, where purities are a random variable determined according to the "normal" and "uniform" methods described in Section 3.2.3.

The decision criterion for a mock sample to provide a sufficient match to the observed sample, and thus to retain an initial set of Schechter parameters for the posterior distribution, is to result in the same number of sources as observed, with line fluxes matching to within the $20 \%$ flux calibration uncertainty. The process is repeated for each of the survey fields, until sufficient accepted samples are generated to accurately define the posterior distribution. The same procedure is repeated for both survey fields combined, requiring that the resulting dataset can be represented by a single, common $\mathrm{CO}$ luminosity function. ${ }^{31}$ In the final step, we merge the results from both purity methods by giving each method equal probability. The

\footnotetext{
31 For this last run, the number of mock sources was allowed to differ by one from the observed sample, which we expect to have a minor impact on the result.
}

resulting model parameter posterior distributions are shown in Figure 6.

As shown in Figures 3 and 6, uncertainties are dominated by the faint-end slope below the "knee," given the sensitivity of the survey. Meanwhile, $L_{\mathrm{CO}}^{\prime *}$ and $\Phi_{\mathrm{CO}}^{*}$ are fairly reliably constrained by the data, with a reasonable agreement within the uncertainties between the two survey fields.

\subsection{2. $\mathrm{CO}(\mathrm{J}=2 \rightarrow 1)$ Luminosity Function}

For estimates of the $\mathrm{CO}(J=2 \rightarrow 1)$ luminosity function, we followed two approaches. The first approach excludes all of the candidates used to construct the $\mathrm{CO}(J=1 \rightarrow 0)$ luminosity function from our search, only leaving confirmed $\operatorname{CO}(J=$ $2 \rightarrow 1$ ) sources and upper limits as available constraints (see Paper I). We further exclude one of the $\operatorname{CO}(J=2 \rightarrow 1)$ sources, AzTEC-3 in the COSMOS field (Riechers et al. 2010, 2014; Capak et al. 2011), because the field was chosen to include this source as a bright "calibrator" for the line search methods. Including this source would likely bias the measurement in its luminosity bin toward high values, under the reasonable assumption that a random $\sim 9 \operatorname{arcmin}^{2}$ field would be unlikely to include a $z>5$ source as luminous as AzTEC-3. As lower and upper bounds on the uncertainties, the 5th and 95th percentiles of the Bayesian posterior for inferring a Poisson rate are adopted in GOODS-North (where sources are detected), and the 68th percentile is adopted as an upper limit for the COSMOS field (where no secure CO $J=2 \rightarrow 1$ detections remain after the exclusion of AzTEC-3). The second approach assumes that all $\mathrm{CO}(J=1 \rightarrow 0)$ candidates that are not independently confirmed could potentially be $\mathrm{CO}(J=2 \rightarrow 1)$ emitters at higher redshifts. Although unlikely, this provides shallower but more detailed upper limits on the $\mathrm{CO}(J=2 \rightarrow 1)$ luminosity function in smaller luminosity bins. Estimates are weighted by purities and corrected for completeness using the "normal" purity method and adopting the upper, 90th percentile bounds as upper limits. The results from both methods are consistent and are shown together in Figure 4.

\subsection{COLDz Cold Gas Density of the Universe}

By integrating the measurements and upper limits obtained on the $\mathrm{CO}(J=1 \rightarrow 0)$ and $\operatorname{CO}(J=2 \rightarrow 1)$ luminosity 


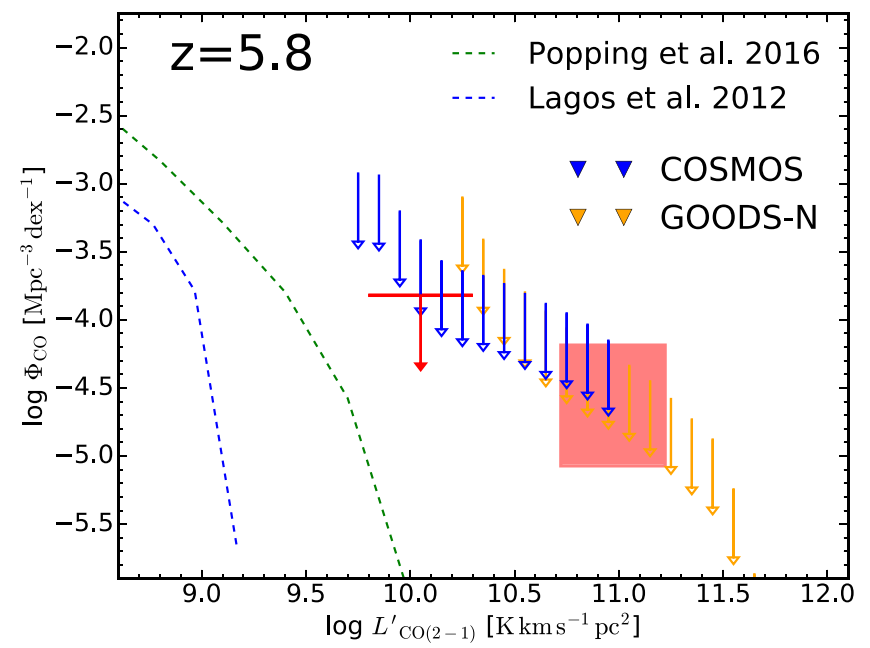

Figure 4. VLA COLDz $\mathrm{CO}(J=2 \rightarrow 1)$ luminosity function at $\langle z\rangle=5.68$ and 5.86 in the COSMOS (blue and red arrows) and GOODS-North (orange arrows and red boxes) fields, respectively. The blue and orange arrows show upper limits under the unlikely assumption that any candidates not independently be confirmed to be $\mathrm{CO}(J=1 \rightarrow 0)$ emission would correspond to $\operatorname{CO}(J=$ $2 \rightarrow 1$ ) emission, for the same binning in $L_{\mathrm{CO}}^{\prime}$ as in Figure 2. Red arrows and boxes consider only independently confirmed $\mathrm{CO}(J=2 \rightarrow 1)$ candidates. The dashed lines show the model predictions.

functions across each of the full redshift intervals, we obtain estimates of the total CO luminosity density per unit volume. For the higher-redshift bin, we use the results from the two methods described above as a direct measurement and as a conservative upper limit, respectively. As in previous work (e.g., Walter et al. 2014; Decarli et al. 2016a), we do not extrapolate the faint end of the luminosity function but instead we only include measurements down to the limit of our survey of $\log \left(L_{\mathrm{CO}}^{\prime} / \mathrm{K} \mathrm{km} \mathrm{s}^{-1} \mathrm{pc}^{2}\right) \simeq 9.5$. Given the consistency of the COLDz data with a flat faint-end slope, and the moderate survey statistics, this assumption is not likely to dominate the uncertainty budget of our measurement but more sensitive observations are required to fully assess the impact of this assumption. ${ }^{32}$ We then convert the measurements of the CO luminosity density to a molecular gas mass density by applying a "standard" conversion factor of $\alpha_{\mathrm{CO}}=$ 3.6 $M_{\odot}\left(\mathrm{K} \mathrm{km} \mathrm{s}^{-1} \mathrm{pc}^{2}\right)^{-1}$ (e.g., Daddi et al. 2010). This choice is motivated by the finding that the majority of the independently confirmed $\mathrm{CO}(J=1 \rightarrow 0)$ emitters (with the exception of the major merger GN19) are consistent with the star-forming galaxy "main sequence" at $z \sim 2-3$. We do not apply a separate correction to $\alpha_{\mathrm{CO}}$ for the $\mathrm{CO}(J=2 \rightarrow 1)$ based measurement because typical $\mathrm{CO}(J=2 \rightarrow 1) / \mathrm{CO}$ $(J=1 \rightarrow 0)$ line brightness temperature ratios for "normal" high-redshift galaxies are of the order $90 \%$ (e.g., Carilli \& Walter 2013) and because the actual $\operatorname{CO}(J=2 \rightarrow 1)$ detections in our survey are all massive dust-obscured starburst galaxies. As such, the implied $\sim 10 \%$ correction required is likely to be sub-dominant to the assumptions made for the choice of $\alpha_{\mathrm{CO}}$ (which also depends on other factors like metallicity; see Bolatto et al. 2013 for a review). When dynamical mass estimates based on spatially resolved

\footnotetext{
32 For reference, integrating our best-fit model Schechter functions down only to $\log \left(L_{\mathrm{CO}}^{\prime} / \mathrm{K} \mathrm{km} \mathrm{s}^{-1} \mathrm{pc}^{2}\right)=10.0$ would result in a $0.07 \mathrm{dex}$ lower median value for the integrated $\mathrm{CO}(J=1 \rightarrow 0)$ luminosity density. Integrating down further to $\log \left(L_{\mathrm{CO}}^{\prime} / \mathrm{K} \mathrm{km} \mathrm{s}^{-1} \mathrm{pc}^{2}\right)=8.0$ or 9.0 would result in $0.044 \mathrm{dex}$ or 0.03 dex higher values, respectively.
}

measurements of individual line candidates become available, we will re-evaluate the choice of $\alpha_{\mathrm{CO}}$. However, we note that the choice of a smaller, "starburst-like" $\alpha_{\mathrm{CO}}$ of order unity would result in significantly lower cold gas density estimates. The resulting measurements of the cold gas density of the universe are shown in Figure 5 and summarized in Table 3.

\section{Discussion}

Due to improved statistics, the COLDz data provide the currently best constraints on the $\mathrm{CO}$ luminosity function at $z \sim 2-3$ and $z \sim 5-7$, and they allow for a measurement of the shape of the CO luminosity function in the $z \sim 2-3$ bin. This provides the perhaps most solid constraints to date on the cosmic density of cold molecular gas in galaxies at these redshifts.

\subsection{Comparison to Previous "Blind" CO Surveys}

\subsubsection{CO Luminosity Function}

The most similar measurements of the $\mathrm{CO}$ luminosity function to COLDz are those in the HDF-N and in the HUDF (ASPECS-Pilot survey) over $\sim 0.5$ and $1 \operatorname{arcmin}^{2}$ size regions, covering the $\mathrm{CO}(J=3 \rightarrow 2)$ line at $\langle z\rangle=2.75$ and $\langle z\rangle=2.61$, respectively (Decarli et al. 2014, 2016a; Walter et al. 2014, 2016). We consider the differences in redshift in these previous works to the $\sim 60 \operatorname{arcmin}^{2} \mathrm{COLDz} \mathrm{CO}(J=$ $1 \rightarrow 0)$ survey $(\langle z\rangle=2.35$ and 2.44 in the COSMOS and GOODS-North fields, respectively) presented here negligible compared to other sources of uncertainty, such that we directly compare these measurements in the following. The difference in line search methods and the luminosity function calculation yield perhaps more conservative uncertainty estimates for the COLDz constraints, although we have confirmed that we would obtain consistent results when adopting the same methods employed in the analysis of the ASPECS-Pilot survey (Decarli et al. 2016a). We thus adopt the measurements and uncertainties from the previous surveys without further modification.

As shown in Figures 2 and 3, we find that the measurements of all three surveys are consistent within the relative uncertainties. There may, however, be tentative evidence that the COLDz measurements are somewhat lower than the ASPECS-Pilot measurements in the best-constrained common luminosity range at $\log \left(L_{\mathrm{CO}}^{\prime} / \mathrm{K} \mathrm{km} \mathrm{s}^{-1} \mathrm{pc}^{2}\right) \simeq 10.2-11.0$ (Figures 2 and 3 ). If real, then this effect may be due to cosmic variance or it could be an indication that $\mathrm{CO}(J=3 \rightarrow 2)$-based surveys preferentially select galaxies with higher gas excitation, such that the $\mathrm{CO}(J=3 \rightarrow 2) / \mathrm{CO}$ $(J=1 \rightarrow 0)$ brightness temperature ratio of $r_{31}=0.42 \pm 0.07$ assumed by Decarli et al. (2016a) to correct for the average gas excitation may be too low (which could then mimic such an effect in principle, depending on the intrinsic shape of the $\mathrm{CO}$ luminosity function). ${ }^{33}$ The latter would be consistent with the finding of a high line ratio limit of $r_{31}>0.7$ for a candidate overlapping between the HDF-N and COLDz surveys (ID19; Decarli et al. 2014), and the lack of $\mathrm{CO}(J=1 \rightarrow 0)$ detections

\footnotetext{
33 For a sample of bright, observed-frame $850 \mu \mathrm{m}$ selected galaxies, Bothwell et al. (2013) find a median $r_{31}$ of $0.52 \pm 0.09$, but while there is source overlap, these galaxies are typically more intensely star-forming than the majority of sources found in the "blind" CO surveys. In addition, the $\sim 20 \% \pm$ $20 \%$ difference in $r_{31}$ is perhaps not sufficient to fully explain the observed effect.
} 

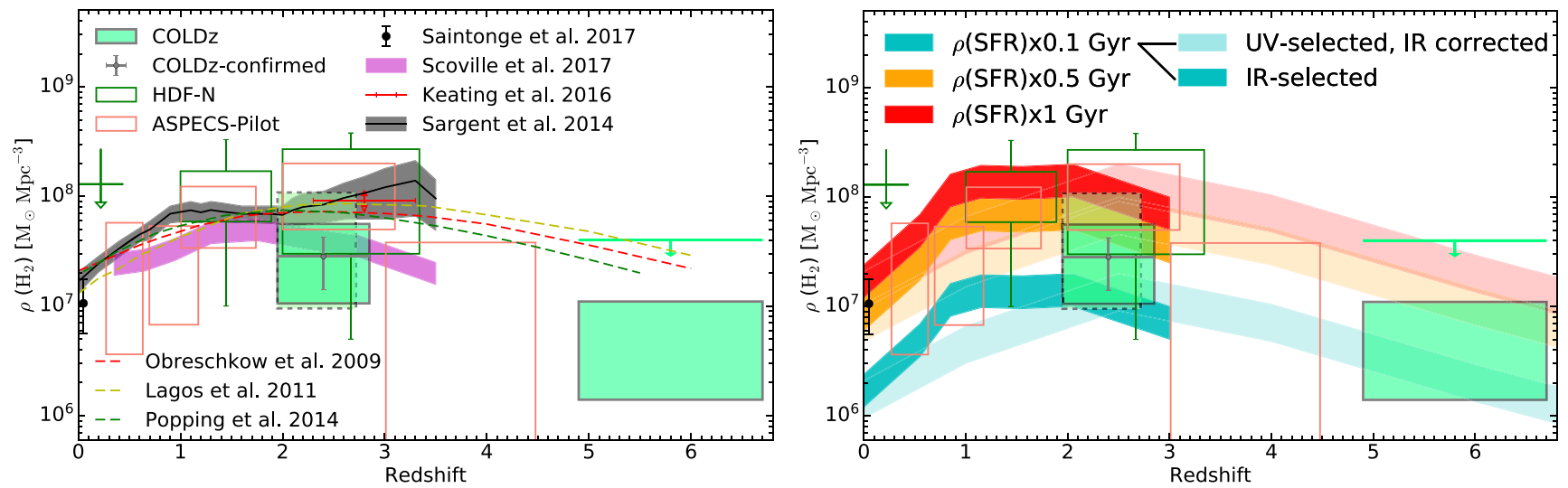

Figure 5. VLA COLDz measurements of the cold gas history of the universe (green boxes), i.e., the co-moving cosmic mass density of cold molecular gas as a function of redshift, showing that the gas density evolves. Vertical sizes indicate the uncertainties in each bin. In the $z \simeq 2-3$ bin, the smaller solid box shows the constraints from both fields combined, and the larger dashed box shows the constraints from the COSMOS field only (both after merging the two purity methods), as an illustration of the impact of field-to-field variations. Assumptions for the measurement and uncertainties in the $z=4.90-6.70$ bin are the same as in Figure 4 . For reference, the gray point shows the measurement obtained when only including independently confirmed candidates, which is fully consistent with the measurement obtained from the complete statistical analysis. Empty green and orange boxes show the constraints from the same surveys as in Figure 2, where different boxes correspond to estimates obtained in different CO transitions (Walter et al. 2014; Decarli et al. 2016a). The black point shows constraints at $z=0$ (Saintonge et al. 2017). Left-hand panel: dashed lines show model predictions (Obreschkow et al. 2009; Lagos et al. 2011; Popping et al. 2014a, 2014b). The gray shaded range shows empirical predictions based on an inversion of the $M_{\text {gas }}-$ SFR relation (e.g., Sargent et al. 2012, 2014; scaled to $\alpha_{\mathrm{CO}}=3.6 M_{\odot}\left(\mathrm{K} \mathrm{km} \mathrm{s}^{-1} \mathrm{pc}^{2}\right)^{-1}$ from its original effective value of $\left.\sim 4.4 M_{\odot}\left(\mathrm{K} \mathrm{km} \mathrm{s}^{-1} \mathrm{pc}^{2}\right)^{-1}\right)$. The magenta range shows estimates based on galaxy stellar mass functions using the dust-based interstellar medium mass scaling method as described by Scoville et al. (2017). None of the measurements are extrapolated to account for the faint end of the molecular gas mass function that remained inaccessible to each survey. The red bar indicates the constraint obtained from intensity mapping by Keating et al. (2016). No uncertainties are shown for this measurement because they are dominated by model assumptions rather than statistical measurement errors. Right-hand panel: same data but also showing the total star formation rate density multiplied by equivalent gas depletion timescales of $0.1,0.5$, and $1.0 \mathrm{Gyr}$, for reference. The lighter shaded regions correspond to star formation rate estimates based on ultraviolet stellar light measurements, with "corrections" for estimated losses due to dust extinction of the ultraviolet light applied. Darker shaded regions correspond to star formation rate estimates based on direct measurements of the dust-obscured stellar light at infrared wavelengths (Bouwens et al. 2016, including infrared-bright sources from Magnelli et al. (2013); see, e.g., Madau \& Dickinson (2014) for further details on uncertainties of the star formation rate density measurements).

Table 3

Cold Gas Density Evolution Measurements from COLDz

\begin{tabular}{lccc}
\hline \hline Redshift Range & $\begin{array}{c}\text { Lower Limit } \\
\text { (5th Percentile) } \\
10^{7} M_{\odot} \mathrm{Mpc}^{-3}\end{array}$ & $\begin{array}{c}\text { Median } \\
(50 \text { th Percentile) } \\
10^{7} M_{\odot} \mathrm{Mpc}^{-3}\end{array}$ & $\begin{array}{c}\text { Upper Limit } \\
\left(\begin{array}{c}\text { 95th Percentile) } \\
10^{7} M_{\odot} \mathrm{Mpc}^{-3}\end{array}\right.\end{array}$ \\
\hline $1.95-2.85$ & 1.1 & 2.7 & 5.6 \\
$1.95-2.72$ & $0.95^{\mathrm{a}}$ & $3.5^{\mathrm{a}}$ & $10.9^{\mathrm{a}}$ \\
$2.03-2.85$ & $0.30^{\mathrm{b}}$ & $1.9^{\mathrm{b}}$ & $7.3^{\mathrm{b}}$ \\
$4.90-6.70$ & 0.14 & 0.47 & 1.1 \\
& & & $4.0^{\mathrm{c}}$ \\
\hline
\end{tabular}

Notes.

${ }^{\text {a }}$ Measurement for the COSMOS field alone, after merging both purity methods.

${ }^{b}$ Measurement for the GOODS-North field alone, after merging both purity methods. These data alone do not fully sample the "knee" of the CO luminosity function.

${ }^{\mathrm{c}}$ Less constraining upper limit obtained when making the (unlikely) assumption that all $\mathrm{CO}(J=1 \rightarrow 0)$ candidates not yet independently confirmed could, in principle, be $\mathrm{CO}(J=2 \rightarrow 1)$ emitters.

for other mid-J CO candidates in the same field (see Paper I). Given that some of these earlier candidates may be spurious, and given the limited statistics of the current surveys and the limited magnitude of the effect, additional data are required to further investigate the relevance of potential selection effects due to CO excitation. In particular, Decarli et al. (2016b) find that some confirmed sources in the ASPECS-Pilot survey appear to show comparatively low $\mathrm{CO}$ excitation, which is in contrast to what would be expected in the case of a $\mathrm{CO}$ excitation-based selection bias. The full, extended ASPECS survey data expected from an ongoing ALMA Large Program will further constrain the contribution of cosmic variance to the observed effect.

\subsubsection{Cold Gas Density of the Universe}

The constraints on the evolution of the cold gas density with redshift resulting from the improved $\mathrm{CO}$ luminosity function measurements provided by COLDz are consistent with those from previous surveys within the relative uncertainties and they extend the range of estimates to earlier cosmic epochs (Figure 5, all $\mathrm{CO}$ surveys assume the same $\alpha_{\mathrm{CO}}$ ). As in the case of the $\mathrm{CO}$ luminosity function constraints, we adopt the measurements and uncertainties from previous works without further modification. Due to differences in the methods used to determine and report uncertainties, caution is advised when comparing the constraints from different surveys at face value. ${ }^{34}$ Previous surveys carried out at 3 and $1 \mathrm{~mm}$ did not provide estimates at $z>4.5$ because these redshifts are only covered in high- $J$ lines, where the estimates of $\mathrm{CO}$ excitation that are necessary to extrapolate the $\mathrm{CO}(J=1 \rightarrow 0)$ luminosity are increasingly uncertain.

The COLDz measurements likely suggest a higher gas density at $z \sim 2-3\left(\rho\left(\mathrm{H}_{2}\right)=0.95-10.9 \times 10^{7} M_{\odot} \mathrm{Mpc}^{-3}\right.$, with

\footnotetext{
${ }^{34}$ For the HDF-N measurements, the lower and upper limits of the boxes shown represent secure detections and all line candidates reported by Walter et al. (2014), respectively, with Poissonian uncertainties due to the number of candidates added as error bars. For the ASPECS-Pilot measurements, box sizes indicate Poissonian errors, with a minor contribution due to flux errors and potential line misidentifications added (Decarli et al. 2016a). Both surveys adopt $1 \sigma$ uncertainty ranges, rather than the more conservative $90 \%$ confidence intervals adopted for the COLDz measurements.
} 
a preferred range of $\left.1.1-5.6 \times 10^{7} M_{\odot} \mathrm{Mpc}^{-3}\right)^{35}$ compared to $z=0 \quad\left(\rho\left(\mathrm{H}_{2}\right)=1.1_{-0.5}^{+0.7} \times 10^{7} M_{\odot} \mathrm{Mpc}^{-3} ;\right.$ Saintonge et al. 2017; see also Keres et al. 2003; Boselli et al. 2014) ${ }^{36}$ by a factor of a few. This finding is consistent with what was reported by the ASPECS team within the relative uncertainties $\left(\rho\left(\mathrm{H}_{2}\right)=4.9-19 \times 10^{7} M_{\odot} \mathrm{Mpc}^{-3} ;\right.$ Decarli et al. 2016a). These measurements are also in agreement with estimates based on galaxy stellar mass functions in COSMOS using the dust-based interstellar medium mass scaling method as described by Scoville et al. (2017). Averaging their data at $z=2.25$ and 2.75 , Scoville et al. suggest $\rho($ ISM $)=$ $3.8 \times 10^{7} M_{\odot} \mathrm{Mpc}^{-3}$ at $z=2.5$. $^{37}$ The latter agrees to within $\sim 30 \%$ with the median value of the COLDz measurement, and within $<10 \%$ with the median value measured in the COSMOS field alone. The COLDz results are also consistent with the constraints obtained from $\mathrm{CO}(J=1 \rightarrow 0)$ intensity mapping experiments at similar redshifts in the GOODS-North field $\left(\rho\left(\mathrm{H}_{2}\right)=9.2_{-3.3}^{+5.9} \times 10^{7} M_{\odot} \mathrm{Mpc}^{-3}\right.$ at $z=2.3-3.3$; Keating et al. 2016). ${ }^{38}$ A comparison of these results is generally valuable because $\mathrm{CO}$ intensity maps may in principle contain signal below the detection threshold of galaxy surveys, but we note that a quantitative comparison of the relative uncertainties is difficult. This is due to the fact that the intensity mapping constraints only measure the second raw moment of the luminosity function and, therefore, cannot distinguish between contributions due to the characteristic luminosity and volume density to the measurement. Furthermore, the detailed interpretation of the nature of the intensity mapping signal in principle relies on assuming a scaling relation between dark matter halo mass and $\mathrm{CO}$ luminosity, which is currently not well constrained at $z \sim 2-3$. We thus do not show formal error bars for this measurement in Figure 5.

The COLDz measurements are also consistent with a decrease in gas density from $z \sim 2-3$ toward $z \sim 5-7\left(\rho\left(\mathrm{H}_{2}\right)=\right.$ $\left.0.14-1.1 \times 10^{7} M_{\odot} \mathrm{Mpc}^{-3}\right)$, possibly to below the present-day value. The redshift evolution of the cold gas history of the universe thus appears qualitatively similar to that of the star formation history of the universe (e.g., Madau \& Dickinson 2014), which is consistent with what is expected if a universal "star formation law" between gas mass and star formation rate (e.g., Carilli \& Walter 2013) already existed at early epochs.

\subsubsection{Gas Depletion Times}

In combination, the cold gas mass and star formation rate density evolution entail information about the evolution

\footnotetext{
35 For reference, the contribution from independently confirmed sources alone is $\rho\left(\mathrm{H}_{2}\right)=(2.8 \pm 1.4) \times 10^{7} M_{\odot} \mathrm{Mpc}^{-3}$, which is consistent with the median value of the total of $2.7 \times 10^{7} \mathrm{M}_{\odot} \mathrm{Mpc}^{-3}$. Paper I also reports a weak $\mathrm{CO}(J=1 \rightarrow 0)$ detection from stacking 34 individually undetected $z=2.0-2.8$ galaxies with stellar masses of $M_{\star}>10^{10} M_{\odot}$ in the GOODSNorth field. Because they are not detected individually, these galaxies are not part of the statistical sample used in this paper. If we were to include these sources, they would contribute an additional $\rho\left(\mathrm{H}_{2}\right) \simeq 0.3 \times 10^{7} M_{\odot} \mathrm{Mpc}^{-3}$ in aggregate.

${ }^{36}$ Here and in Figure 5, we adopt $\alpha_{\mathrm{CO}}=3.6 M_{\odot}\left(\mathrm{K} \mathrm{km} \mathrm{s}^{-1} \mathrm{pc}^{2}\right)^{-1}$ for consistency. Adopting $\alpha_{\mathrm{CO}}=6.5 M_{\odot}\left(\mathrm{K} \mathrm{km} \mathrm{s}^{-1} \mathrm{pc}^{2}\right)^{-1}$ as used by Keres et al. (2003) would result in a factor of 1.8 higher $\rho\left(\mathrm{H}_{2}\right)$.

37 Here and in Figure 5, we adopt $\alpha_{\mathrm{CO}}=3.6 M_{\odot}\left(\mathrm{K} \mathrm{km} \mathrm{s}^{-1} \mathrm{pc}^{2}\right)^{-1}$ for consistency. Adopting $\alpha_{\mathrm{CO}}=6.5 M_{\odot}\left(\mathrm{K} \mathrm{km} \mathrm{s}^{-1} \mathrm{pc}^{2}\right)^{-1}$ as used by Scoville et al. (2017) would result in a factor of 1.8 higher $\rho\left(\mathrm{H}_{2}\right)$.

${ }^{38}$ Here and in Figure 5, we adopt $\alpha_{\mathrm{CO}}=3.6 M_{\odot}\left(\mathrm{K} \mathrm{km} \mathrm{s}^{-1} \mathrm{pc}^{2}\right)^{-1}$ for consistency. Adopting $\alpha_{\mathrm{CO}}=4.3 M_{\odot}\left(\mathrm{K} \mathrm{km} \mathrm{s}^{-1} \mathrm{pc}^{2}\right)^{-1}$ as used by Keating et al. (2016) would result in a factor of 1.2 higher $\rho\left(\mathrm{H}_{2}\right)$.
}

of galaxy gas depletion times as a function of redshift. ${ }^{39}$ As shown in Figure 5, simply multiplying the total star formation rate density (Bouwens et al. 2016) by a characteristic gas depletion timescale (which, to first order, represents the ratio between molecular gas mass and star formation rate, $M_{\mathrm{H}_{2}} / \mathrm{SFR}$ ) of several hundred million years provides a reasonable match to the cold gas density relation at all redshifts currently probed within the uncertainties, although the data may tentatively prefer shorter depletion times toward higher redshifts. At $z=0$, a characteristic gas depletion timescale in the range of $\tau_{\text {dep }}^{\text {ch }}=0.5-1$ Gyr is preferred with the adopted $\alpha_{\mathrm{CO}}$ conversion factor (see also discussion by Saintonge et al. 2017). Based on the COLDz measurements of $\rho\left(\mathrm{H}_{2}\right)$ at $z=2.4$ and adopting $\rho(\mathrm{SFR})=0.15 \pm 0.05 M_{\odot} \mathrm{yr}^{-1} \mathrm{Mpc}^{-3}$ (e.g., Madau $\&$ Dickinson 2014), we find a characteristic gas depletion timescale of $\tau_{\text {dep }}^{\text {ch }}=70-750 \mathrm{Myr}$ with $90 \%$ confidence, with a median value of $200 \pm 70 \mathrm{Myr}$. Since the star formation rate density relation includes significantly less luminous galaxies than probed by current blind $\mathrm{CO}$ surveys, this may either indicate that low-luminosity galaxies below our $L_{\text {CO }}^{\prime}$ detection limit do not contribute dominantly to the total cold gas density (perhaps implying that the faint-end slope of the $\mathrm{CO}$ luminosity function is not steeply rising toward lower $L_{\mathrm{CO}}^{\prime}$ ) or that the characteristic gas depletion timescales are longer than 200-500 Myr when averaged over the entire galaxy population. Assuming substantially shorter gas depletion timescales (or, high star formation efficiencies) appears to be inconsistent with the data, unless the characteristic $\alpha_{\mathrm{CO}}$ conversion factor is substantially lower than assumed. The COLDz measurements of $\rho\left(\mathrm{H}_{2}\right)$ at $z=5.8$ are consistent with characteristic gas depletion timescales of $\tau_{\text {dep }}^{\text {ch }}>100 \mathrm{Myr}$, with a factor of a few higher values allowed by the data within the uncertainties. Although not a unique conclusion based on the COLDz data given the remaining uncertainties, a shortening in gas depletion times despite the observed increase in cold molecular gas content in star-forming galaxies toward higher redshift would be consistent with similar findings based on targeted studies of $\mathrm{CO}(J=3 \rightarrow 2)$ emission and dust-based interstellar medium mass estimates (e.g., Genzel et al. 2015; Scoville et al. 2017), and thus with an effective increase in star formation efficiency (i.e., SFR per unit $M_{\mathrm{H}_{2}}$ ) toward higher redshifts.

\subsection{Comparison to Model Predictions}

\subsubsection{CO Luminosity Function}

Given the consistency between the COLDz data and previous surveys, we compare the new $\mathrm{CO}$ luminosity function measurements to predictions based on semi-analytical models (Lagos et al. 2012; Popping et al. 2016) and empirical estimates based on the infrared luminosity function of Herschel-selected galaxies under the assumption of a "star formation law" (Vallini et al. 2016; Figures 2-4; see, e.g., Lagos et al. 2015; Davé et al. 2017; Xie et al. 2017 for additional model predictions).

The measurements at $z \sim 2-3$ appear to be inconsistent with the semi-analytical predictions (see Decarli et al. 2016a for a detailed comparison of both models), which place the characteristic luminosity $L_{\text {CO }}^{\prime *}$ ("knee") of the luminosity function at significantly lower luminosities than observed.

\footnotetext{
39 The analysis presented here concerns the redshift evolution of gas depletion times, and thus does not further consider the potential range of values expected for different galaxy populations that contribute to the signal.
} 
This is consistent with the excess of bright sources compared to the predictions seen in the ASPECS-Pilot data alone in some luminosity bins (Decarli et al. 2016a), although the trend becomes clearer at the higher statistical significance of the COLDz measurements-showing a significantly (by one to two orders of magnitude) higher characteristic luminosity than that observed at $z \sim 0$ (e.g., Keres et al. 2003; Boselli et al. 2014; Saintonge et al. 2017). These predictions also prefer a strong contribution from faint sources, which is not preferred by the data but agrees within the considerable uncertainties of the measurements at low luminosities. Qualitatively, the underprediction in the number of luminous $\mathrm{CO}$ emitters may be related to the finding that semi-analytical models tend to underpredict the star formation rates of galaxies on the starforming main sequence at similar redshifts (see, e.g., review by Somerville \& Davé 2015).

At $z \sim 5-7$, the excess of bright sources compared to the semi-analytical predictions appears to be even more pronounced than at lower redshifts but we caution that the most constraining measurement is based on a small number of sources only, and thus needs to be put on a firmer statistical footing. Meanwhile, the observations at $z \sim 2-3$ appear to be consistent with Vallini et al.'s (2016) empirical predictions, and thus with what is expected from estimates of dust-obscured star formation activity at high redshift based on infrared luminosity functions.

\subsubsection{Cold Gas Density of the Universe}

The observational constraints on the evolution of the cold gas density with redshift remain in agreement with both semianalytical (Obreschkow et al. 2009; Lagos et al. 2011, 2012; Popping et al. 2014a, 2014b) and empirical (Sargent et al. 2012 , 2014) model predictions at $z \sim 2-3$. Since most of the semi-analytical models include a varying $\alpha_{\mathrm{CO}}$ between individual galaxies, a simple interpretation of the consistency - despite the disagreement in the luminosity function estimates - remains challenging. In addition, the predictions do not account for the sensitivity limits of the $\mathrm{CO}$ surveys, or uncertainties due to cosmic variance. Given the differences in the CO luminosity functions between models and observations at high $z$, this effect could in principle lead to up to a factor of a few difference in the corresponding gas densities at high redshift. However, as discussed in Section 3.4, the impact of the sensitivity limits, which would bias the measurements toward lower values, appears to be relatively minor based on the preferred model Schechter function fits to the COLDz data. Meanwhile, cosmic variance due to large-scale structure in the distribution of gas-rich galaxies (which is assumed to be uniform in our analysis) could bias the measurements either low or high. To obtain an approximate estimate of systematic uncertainties introduced by cosmic variance, we follow the prescription by Driver \& Robotham (2010), based on the distribution of galaxies near the characteristic stellar mass at a given redshift. ${ }^{40}$ Including both Poisson uncertainty and cosmic variance scaled to the volume of the COLDz survey, we find a sample variance uncertainty of $\sim 30 \%-40 \%$ and

\footnotetext{
${ }^{40}$ This method is based on a generalized expression (their Equation (4)) calibrated through an examination of galaxies within $\pm 1 \mathrm{mag}$ of the characteristic optical magnitude out to $z \sim 0.1$ in the Sloan Digital Sky Survey (SDSS), which represent the most common galaxies at a given redshift. See cosmocalc.icrar.org for additional details. The field sizes and $\mathrm{CO}(J=1 \rightarrow 0)$ redshift ranges in Table 1 are used for all calculations.
}

$\sim 25 \%$ for the COSMOS and GOODS-North fields, respectively. Alternatively, adapting results based on models of the evolution of the most massive galaxies (i.e., $M_{\star}>10^{11} M_{\odot}$ ) by Moster et al. $(2011)^{41}$ yields an estimated uncertainty of $\sim 40 \%-50 \%$ based on cosmic variance alone for the smaller COSMOS field. These estimates are consistent with those found from more detailed calculations based on the IllustrisTNG simulations (of order $\sim 30 \%-50 \%$ in the $z \sim 2-3$ bin for both fields combined; G. Popping 2018, private communication). All of the estimates appear to suggest that uncertainties due to cosmic variance are sub-dominant to other sources of uncertainty, given the large volume of the COLDz survey due to the broad range in redshift covered and the comparatively large field size. This is also consistent with the broad distribution in redshift of the confirmed $\mathrm{CO}$ emitters and candidates in the COLDz survey volume (see, e.g., Paper I, Figure 3). ${ }^{42}$ In any case, if we were to conservatively correct down the model predictions by factors of $\sim 1.5-2$ to account for the combined effects of sensitivity limits and cosmic variance, they would in fact move close to the median $\rho\left(\mathrm{H}_{2}\right)$ implied by the COLDz measurements. Taken at face value, the apparent agreement between the model predictions and $\mathrm{COLDz}$ data could indicate that there is no significant contribution-or at least, no dominant contribution-from sources far below the COLDz detection limit, such that steeply rising faint-end slopes of the $\mathrm{CO}$ luminosity function toward lower $L_{\mathrm{CO}}^{\prime}$ may be disfavored. This would also be consistent with the agreement between the COLDz measurement and intensity mapping constraints.

If true, this could be related to lower metallicities toward fainter, low-mass galaxies, leading to disproportionally low CO luminosity per unit molecular gas mass (e.g., Genzel et al. 2012; Bolatto et al. 2013). Although not a unique explanation, this would be consistent with the finding of a lower median redshift of galaxies with low submillimeter continuum fluxes compared to brighter ones (implying low dust masses, and thus likely low gas masses; Aravena et al. 2016) and with the apparent finding of a low dust content in lower stellar mass galaxies at $z>2$ (Bouwens et al. 2016). This would also be consistent with the finding that we do not detect $\mathrm{CO}(J=2 \rightarrow 1)$ emission from several known, modestly massive and star-forming Lyman-break galaxies at $z \sim$ 5.2-5.3 in our survey area (Paper I; see also Capak et al. 2011; Walter et al. 2012; Riechers et al. 2014), which is compatible with a perhaps elevated $\alpha_{\mathrm{CO}}$ due to lower metallicity. All of the confirmed $z>5 \mathrm{COLDz}$ detections are massive, dust-obscured starburst galaxies with likely high metallicity.

The observational constraints at $z \sim 5-7$ are also in agreement with the model predictions, albeit lower than the Obreschkow et al.'s and Lagos et al.'s models unless some unconfirmed sources (which are taken into consideration for the upper limit shown) contribute to the signal. They are less secure than in the lower-redshift bin due to more limited

\footnotetext{
41 For our estimate, we have made use of the predictions provided for the $\mathrm{H}-\mathrm{UDF}$ because this field has a similar area as the COLDz COSMOS field. We have assumed $\langle z\rangle=2.35$ and $\Delta z=0.8$ in our calculations, and we adopt the values found for the $M_{\star}=10^{11.0}-10^{11.5} M_{\odot}$ bin.

${ }^{42}$ The COSMOS field contains the AzTEC-3 protocluster region at $z=5.3$ (e.g., Riechers et al. 2010, 2014; Capak et al. 2011), and thus is biased in principle. However, only upper limits are reported in this field for the corresponding redshift bin, such that this does not impact the reported measurements.
} 
statistics and because it is not currently possible to measure the characteristic CO luminosity at these redshifts, such that the fraction of the total cold gas density recovered down to the sensitivity limit of the survey is less certain than at lower redshifts. Nonetheless, this finding appears to be consistent with the assumption of an evolving $\alpha_{\mathrm{CO}}$ due to lower metallicity in fainter galaxies and toward higher redshifts, resulting in a steep drop in the gas volume density as traced by $\mathrm{CO}$ emission. Further observations are required to investigate if the drop in $\mathrm{H}_{2}$ density toward very high redshift is as steep as observed in $\mathrm{CO}$, or if the effect is enhanced due to metallicity affecting the strength of the $\mathrm{CO}$ signal. ${ }^{43}$

\section{Conclusions}

We have used "blind" molecular line scans over $\sim 60 \mathrm{arcmin}^{2}$ in the COSMOS and GOODS-North survey fields that were taken as part of the VLA COLDz survey (Paper I) to measure the shape of the CO luminosity function at $z \sim 2-3$ and to constrain it at $z \sim 5-7$, utilizing $\mathrm{CO}(J=1 \rightarrow 0)$ and $\mathrm{CO}(J=2 \rightarrow 1)$ emission line galaxy candidates. We also provide constraints on the evolution of the cosmic molecular gas density out to $z \sim 7$. We compare our findings to previous $\sim 0.5$ and $1 \operatorname{arcmin}^{2}$ surveys in the HDF-N and the H-UDF (ASPECS-Pilot) in higher- $J$ CO lines (Decarli et al. 2014; Walter et al. 2016), estimates based on galaxy stellar mass functions in COSMOS scaled using dust-based interstellar medium mass estimates (Scoville et al. 2017), and a CO intensity mapping study in GOODS-North (Keating et al. 2016), finding broad agreement within the relative uncertainties. The COLDz data provide the first solid measurement of the shape of the $\mathrm{CO}$ luminosity function at $z \sim 2-3$, reaching below its "knee," and the first significant constraints at $z \sim 5-7$. The characteristic CO luminosity at $z \sim 2-3$ appears to be one to two orders of magnitude higher than at $z=0$ (Keres et al. 2003; Saintonge et al. 2017), which is consistent with the idea that the dominant star-forming galaxy populations $\sim 10$ billion years ago were significantly more gas-rich compared to the present day. We also independently confirm an observed apparent excess of the space density of bright CO-emitting sources at high redshift compared to semianalytical predictions, even though our findings are consistent with empirical predictions based on the infrared luminosity function and observed star formation rates of distant galaxies.

By integrating the $\mathrm{CO}$ luminosity functions down to the sensitivity limit of our survey, we obtain robust estimates of the volume density of cold gas in galaxies at high redshift. Our measurement is consistent with a factor of a few increase from $z \sim 0$ to $z \sim 2-3$ and a decrease toward $z \sim 5-7$ by about an order of magnitude (which may be less steep in practice if metallicity has an increasing effect on CO-based measurements toward the highest redshifts). This is consistent with semi-analytical and empirical model predictions and previous constraints from the ASPECS-Pilot survey (Decarli et al. 2016a), and is also consistent with previous findings of increased gas fractions at $z>1-2$ (e.g., Daddi et al. 2010;

\footnotetext{
$\overline{43}$ The strength of the $\mathrm{CO}$ signal may also be reduced at the highest redshifts due to the increased temperature of the cosmic microwave background, relative to which the cold gas emission is detected. The importance of this effect strongly depends on the excitation of the gas traced by $\mathrm{CO}$, particularly the kinetic gas temperature (e.g., da Cunha et al. 2013).
}

Tacconi et al. 2013, 2018; Scoville et al. 2017). The overall shape of the cosmic gas density evolution resembles that of the star formation history of the universe, which is consistent with an underlying "star formation law" relation that holds out to the highest measured redshifts. This suggests that the star formation history, to the first order, follows the evolution of the molecular gas supply in galaxies, as regulated by the gas accretion efficiency and feedback processes. A more direct comparison of the star formation rate and cold gas density relations as a function of cosmic time holds critical information about the true gas depletion timescales, and thus the gas accretion rates required to maintain the ongoing buildup of stellar mass. The data appear to be broadly consistent with a characteristic gas depletion timescale of several hundred million years, but there may be tentative evidence for a shortening in gas depletion times despite the observed increase in cold molecular gas content in star-forming galaxies toward higher redshift. This finding would be consistent with previous, targeted investigations based on $\mathrm{CO}(J=3 \rightarrow 2)$ and dust-based interstellar medium mass estimates (e.g., Genzel et al. 2015; Scoville et al. 2017), and thus with an effective increase in star formation efficiency in the dominant star-forming galaxy populations toward higher redshifts.

While COLDz is currently the largest survey of its kind, the size of the volume probed and the number of line candidates found implies that larger areas need to be surveyed to a greater depth in the future to more clearly address the effects of cosmic variance and to reduce the error budget due to Poissonian fluctuations. Such studies will be made possible with large investments of observing time at the VLA and ALMA in the coming years, but will remain time intensive at least until the next large leap in capabilities will become available following the construction of the Next Generation Very Large Array (ngVLA; e.g., Bolatto et al. 2017; Selina et al. 2018).

The authors thank Amélie Saintonge for sharing her updated Schechter fits to the local CO luminosity function prior to publication, Rychard Bouwens for sharing the data necessary to create Figure 5, and Gergö Popping for helpful input on the comparison of our results to models, and for providing some new and updated model results. We also thank the anonymous referee for a helpful report. D.R. and R.P. acknowledge support from the National Science Foundation under grant number AST-1614213 to Cornell University. J.A.H. acknowledges support of the VIDI research program with project number 639.042.611, which is (partly) financed by the Netherlands Organisation for Scientific Research (NWO). I.R.S. acknowledges support from the ERC Advanced Grant DUSTYGAL (321334), STFC (ST/P000541/1), and a Royal Society/ Wolfson Merit Award. The National Radio Astronomy Observatory is a facility of the National Science Foundation operated under cooperative agreement by Associated Universities, Inc.

Facility: VLA.

\section{Appendix A}

\section{Luminosity Function Constraints: Tabulated Results}

For reference, in this appendix we include the measured ranges of the $\mathrm{CO}$ luminosity function from the COLDz $\mathrm{CO}(J=1 \rightarrow 0)$ data at $\langle z\rangle=2.4 \quad$ (Table 4) and the $\mathrm{CO}(J=2 \rightarrow 1)$ data at $\langle z\rangle=5.8$ (Tables 5 and 6 ), as utilized 
Table 4

Measured Ranges of the $\mathrm{CO}(J=1 \rightarrow 0)$ Luminosity Function at $z \sim 2.4$ from the COLDz Data (5th and 95th Percentiles)

\begin{tabular}{|c|c|c|c|c|c|}
\hline $\begin{array}{l}\log \left(L_{\mathrm{CO}}^{\prime}\right) \text { bin } \\
\left(\mathrm{K} \mathrm{km} \mathrm{s}^{-1} \mathrm{pc}^{2}\right)\end{array}$ & $\begin{array}{l}\text { COSMOS "uniform"a } \\
\quad\left(\mathrm{Mpc}^{-3} \mathrm{dex}^{-1}\right)\end{array}$ & $\begin{array}{l}\text { COSMOS "normal"a } \\
\left(\mathrm{Mpc}^{-3} \mathrm{dex}^{-1}\right)\end{array}$ & $\begin{array}{l}\text { GOODS-N "uniform"” } \\
\left(\mathrm{Mpc}^{-3} \mathrm{dex}^{-1}\right)\end{array}$ & $\begin{array}{c}\text { GOODS-N "normal” } \\
\left(\mathrm{Mpc}^{-3} \mathrm{dex}^{-1}\right)\end{array}$ & $\begin{array}{l}\text { Combined Fields "merged" } \\
\left(\mathrm{Mpc}^{-3} \mathrm{dex}^{-1}\right)\end{array}$ \\
\hline $9.5-10.0$ & $-4.04,-2.55$ & $-3.77,-2.21$ & $\ldots$ & $\ldots$ & $-4.04,-2.21$ \\
\hline $9.6-10.1$ & $-4.08,-3.03$ & $-3.83,-2.69$ & $\ldots$ & $\ldots$ & $-4.08,-2.69$ \\
\hline $9.8-10.3$ & $-4.19,-3.47$ & $-3.92,-3.12$ & $\ldots$ & $\cdots$ & $-4.19,-3.12$ \\
\hline $9.9-10.4$ & $-4.23,-3.44$ & $-3.96,-3.24$ & $\ldots$ & $\ldots$ & $-4.23,-3.24$ \\
\hline $10.0-10.5$ & $-4.26,-3.38$ & $-3.98,-3.26$ & $-4.25,-3.37$ & $-3.97,-3.00$ & $-4.10,-3.32$ \\
\hline $10.3-10.8$ & $-3.62,-3.36$ & $-3.60,-3.29$ & $-4.39,-3.92$ & $-4.11,-3.62$ & $-3.69,-3.39$ \\
\hline $10.4-10.9$ & $-3.65,-3.41$ & $-3.63,-3.34$ & $-4.47,-4.01$ & $-4.20,-3.74$ & $-3.73,-3.45$ \\
\hline $10.5-11.0$ & $-3.85,-3.45$ & $-3.77,-3.40$ & $-4.59,-4.11$ & $-4.31,-3.86$ & $-3.98,-3.60$ \\
\hline $10.6-11.1$ & $-3.96,-3.49$ & $-3.96,-3.48$ & $-4.71,-4.21$ & $-4.43,-3.97$ & $-4.10,-3.70$ \\
\hline $10.7-11.2$ & $-5.23,-3.69$ & $-4.90,-3.69$ & $-4.82,-4.31$ & $-4.56,-4.07$ & $-4.79,-4.11$ \\
\hline $10.8-11.3$ & $<(-8.00),-3.95$ & $<(-8.00),-3.90$ & $-4.96,-4.41$ & $-4.73,-4.19$ & $-5.00,-4.25$ \\
\hline $11.3-11.8$ & $\ldots$ & $\cdots$ & $<(-8.00),-4.82$ & $<(-8.00),-4.82$ & $<(-8.00),-4.82$ \\
\hline $11.4-11.9$ & $\ldots$ & $\ldots$ & $<(-8.00),-5.96$ & $<(-8.00),-5.56$ & $<(-8.00),-5.56$ \\
\hline
\end{tabular}

Notes. $\log \left(L_{\mathrm{CO}}^{\prime}\right)$ bins in boldface indicate the spacing of statistically independent steps.

a Given as $\log \left(\Phi_{\mathrm{CO}}\right)$. Based on "uniform," "normal," and merged (last column) purity uncertainty estimates as described in Section 3.

Table 5

Measured Ranges of the $\mathrm{CO}(J=2 \rightarrow 1)$ Luminosity Function at $z \sim 5.8$ from the COLDz data (90th Percentile Upper Limits)

\begin{tabular}{lcc}
\hline \hline $\begin{array}{l}\log \left(L_{\mathrm{CO}}^{\prime}\right) \text { bin } \\
\left(\mathrm{K} \mathrm{km} \mathrm{s}^{-1} \mathrm{pc}^{2}\right)\end{array}$ & $\begin{array}{c}\mathrm{COSMOS}^{\mathrm{a}} \\
\left(\mathrm{Mpc}^{-3} \mathrm{dex}^{-1}\right)\end{array}$ & $\begin{array}{c}\mathrm{GOODS}-\mathrm{N}^{\mathrm{a}} \\
\left(\mathrm{Mpc}^{-3} \mathrm{dex}^{-1}\right)\end{array}$ \\
\hline $\mathbf{9 . 5 - 1 0 . 0}$ & $<(-2.92)$ & $\ldots$ \\
$9.6-10.1$ & $<(-2.94)$ & $\ldots$ \\
$9.7-10.2$ & $<(-3.20)$ & $\ldots$ \\
$9.8-10.3$ & $<(-3.42)$ & $\ldots$ \\
$9.9-10.4$ & $<(-3.57)$ & $\ldots$ \\
$\mathbf{1 0 . 0}-\mathbf{1 0 . 5}$ & $<(-3.64)$ & $<(-3.10)$ \\
$10.1-10.6$ & $<(-3.68)$ & $<(-3.41)$ \\
$10.2-10.7$ & $<(-3.74)$ & $<(-3.63)$ \\
$10.3-10.8$ & $<(-3.81)$ & $<(-3.80)$ \\
$10.4-10.9$ & $<(-3.88)$ & $<(-4.06)$ \\
$\mathbf{1 0 . 5}-\mathbf{1 1 . 0}$ & $<(-3.95)$ & $<(-4.16)$ \\
$10.6-11.1$ & $<(-4.03)$ & $<(-4.25)$ \\
$10.7-11.2$ & $<(-4.15)$ & $<(-4.34)$ \\
$10.8-11.3$ & $\ldots$ & $<(-4.45)$ \\
$10.9-11.4$ & $\ldots$ & $<(-4.58)$ \\
$\mathbf{1 1 . 0 - 1 1 . 5}$ & $\ldots$ & $<(-4.73)$ \\
$11.1-11.6$ & $\ldots$ & $<(-4.88)$ \\
$11.2-11.7$ & $\ldots$ & $<(-5.24)$ \\
$11.3-11.8$ & $\ldots$ & $<(-5.87)$ \\
$11.4-11.9$ & $\ldots$ & \\
\hline
\end{tabular}

Notes. $\log \left(L_{\mathrm{CO}}^{\prime}\right)$ bins in boldface indicate the spacing of statistically independent steps.

a Given as $\log \left(\Phi_{\mathrm{CO}}\right)$. Based on "normal" purity uncertainty estimates as described in Section 3.
Table 6

$\mathrm{CO}(J=2 \rightarrow 1)$ Luminosity Function at $z \sim 5.8$ from the COLDz Data Based on Confirmed Sources Only (Red Symbols in Figure 4)

\begin{tabular}{lcc}
\hline \hline $\begin{array}{l}\log \left(L_{\mathrm{CO}}^{\prime}\right) \text { bin } \\
\left(\mathrm{K} \mathrm{km} \mathrm{s}^{-1} \mathrm{pc}^{2}\right)\end{array}$ & $\begin{array}{c}\mathrm{COSMOS}^{\mathrm{a}} \\
\left(\mathrm{Mpc}^{-3} \mathrm{dex}^{-1}\right)\end{array}$ & $\begin{array}{c}\text { GOODS-N } \\
\left(\mathrm{Mpc}^{-3} \mathrm{dex}^{-1}\right)\end{array}$ \\
\hline $9.8-10.3$ & $<(-3.82)$ & $\cdots$ \\
$10.7-11.2$ & $\cdots$ & $-5.07,-4.19$ \\
\hline
\end{tabular}

Note.

${ }^{\mathrm{a}}$ Given as $\log \left(\Phi_{\mathrm{CO}}\right)$. See Section 3.3.2 for details.

in Figure 2-4. The $\log \left(L_{\mathrm{CO}}^{\prime}\right)$ bins are 0.5 dex wide and given in steps of $0.1 \mathrm{dex}$, such that every 5 th bin is statistically independent.

\section{Appendix B \\ Luminosity Function Modeling: Further Details}

In this appendix, we show the corner plots of the Schechter model parameter posterior distribution from fitting the $\operatorname{COLDz} \mathrm{CO}(J=1 \rightarrow 0)$ luminosity function with the $\mathrm{ABC}$ method (Figure 6). The adopted prior ranges are $\log \left(L_{\mathrm{CO}}^{\prime} /\right.$ $\left.\mathrm{K} \mathrm{km} \mathrm{s}^{-1} \mathrm{pc}^{2}\right)=9.5$ to $11.5, \log \left(\Phi_{\mathrm{CO}} / \mathrm{Mpc}^{-3} \mathrm{dex}^{-1}\right)=-5$ to -2.5 , and $\alpha=-1$ to 1 . 

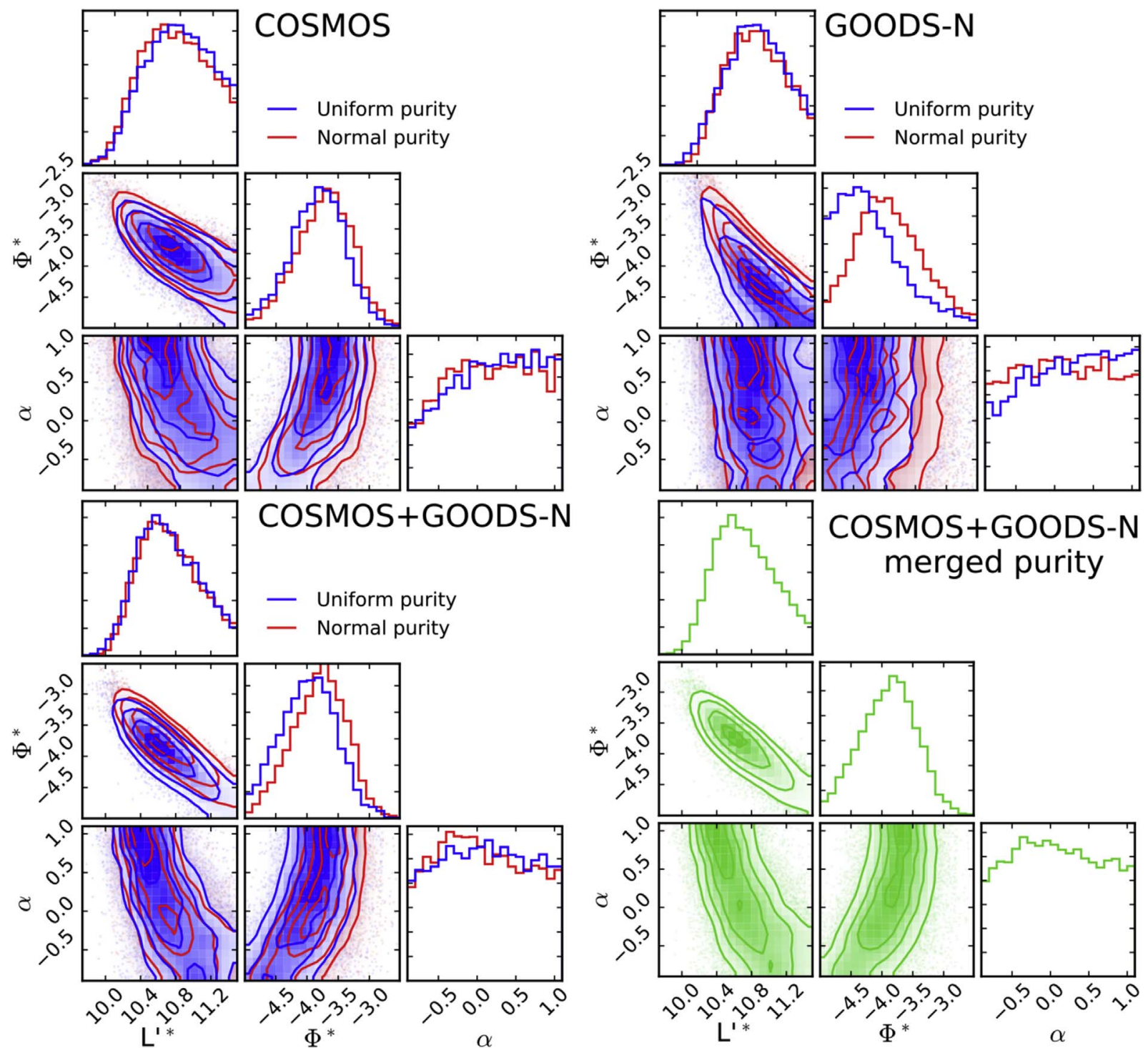

Figure 6. Corner plots of the Schechter model parameter posterior distribution from fitting the $\mathrm{CO}(J=1 \rightarrow 0)$ luminosity function with the ABC method. Contour levels correspond to $0.5,1.0,1.5$, and $2.0 \sigma$ in two-dimensions. Top: parameters found when fitting results obtained with the "uniform" (blue) and "normal" (red) purity methods for the COLDz COSMOS (left-hand) and GOODS-North (right-hand) fields, respectively. Bottom: same as found when combining the constraints from both fields, before (left-hand) and after (right-hand) merging the two methods used to calculate purities, respectively.

\section{ORCID iDs}

Dominik A. Riechers (i) https://orcid.org/0000-00019585-1462

Riccardo Pavesi (1) https://orcid.org/0000-0002-2263-646X Chelsea E. Sharon (i) https://orcid.org/0000-0002-6250-5608 Jacqueline A. Hodge (1) https://orcid.org/0000-0001-6586-8845 Roberto Decarli (10 https://orcid.org/0000-0002-2662-8803 Fabian Walter (1) https://orcid.org/0000-0003-4793-7880 Christopher L. Carilli (i) https://orcid.org/0000-0001-6647-3861 Manuel Aravena (i) https://orcid.org/0000-0002-6290-3198 Elisabete da Cunha (i) https://orcid.org/0000-0001-9759-4797 Emanuele Daddi (1) https://orcid.org/0000-0002-3331-9590 Mark Dickinson (10) https://orcid.org/0000-0001-5414-5131 Ian Smail (1) https://orcid.org/0000-0003-3037-257X Rob J. Ivison (10 https://orcid.org/0000-0001-5118-1313 Mark Sargent (1) https://orcid.org/0000-0003-1033-9684 Nicholas Z. Scoville (i) https://orcid.org/0000-0002-0438-3323

\section{References}

Aravena, M., Carilli, C. L., Salvato, M., et al. 2012, MNRAS, 426, 258 Aravena, M., Decarli, R., Walter, F., et al. 2016, ApJ, 833, 68 Aravena, M., Hodge, J. A., Wagg, J., et al. 2014, MNRAS, 442, 558 Bennett, C. L., Larson, D., Weiland, J. L., \& Hinshaw, G. 2014, ApJ, 794, 135

Bolatto, A. D., Chatterjee, S., Casey, C. M., et al. 2017, arXiv:1711.09960 Bolatto, A. D., Warren, S. R., Leroy, A. K., et al. 2015, ApJ, 809, 175 Bolatto, A. D., Wolfire, M., \& Leroy, A. K. 2013, ARA\&A, 51, 207 Boselli, A., Cortese, L., Boquien, M., et al. 2014, A\&A, 564, A66 Bothwell, M. S., Smail, I., Chapman, S. C., et al. 2013, MNRAS, 429, 3047 Bouché, N., Dekel, A., Genzel, R., et al. 2010, ApJ, 718, 1001 Bouwens, R. J., Aravena, M., Decarli, R., et al. 2016, ApJ, 833, 72 Cameron, E., \& Pettitt, A. N. 2012, MNRAS, 425, 44 Capak, P. L., Riechers, D., Scoville, N. Z., et al. 2011, Natur, 470, 233

Carilli, C. L., \& Walter, F. 2013, ARA\&A, 51, 105

da Cunha, E., Groves, B., Walter, F., et al. 2013, ApJ, 766, 13

Daddi, E., Bournaud, F., Walter, F., et al. 2010, ApJ, 713, 686

Daddi, E., Dannerbauer, H., Liu, D., et al. 2015, A\&A, 577, A46

Davé, R., Finlator, K., \& Oppenheimer, B. D. 2012, MNRAS, 421, 98 
Davé, R., Rafieferantsoa, M. H., Thompson, R. J., \& Hopkins, P. F. 2017, MNRAS, 467, 115

Decarli, R., Walter, F., Aravena, M., et al. 2016a, ApJ, 833, 69

Decarli, R., Walter, F., Aravena, M., et al. 2016b, ApJ, 833, 70

Decarli, R., Walter, F., Carilli, C., et al. 2014, ApJ, 782, 78

Driver, S. P., \& Robotham, A. S. G. 2010, MNRAS, 407, 2131

Faucher-Giguère, C.-A., Kereš, D., \& Ma, C.-P. 2011, MNRAS, 417, 2982

Foreman-Mackey, D., Hogg, D. W., Lang, D., \& Goodman, J. 2013, PASP, 125,306

Genel, S., Bouché, N., Naab, T., Sternberg, A., \& Genzel, R. 2010, ApJ, 719, 229

Genzel, R., Tacconi, L. J., Combes, F., et al. 2012, ApJ, 746, 69

Genzel, R., Tacconi, L. J., Lutz, D., et al. 2015, ApJ, 800, 20

Harrington, K. C., Yun, M. S., Magnelli, B., et al. 2018, MNRAS, 474, 3866

Ishida, E. E. O., Vitenti, S. D. P., Penna-Lima, M., et al. 2015, A\&C, 13, 1

Ivison, R. J., Papadopoulos, P. P., Smail, I., et al. 2011, MNRAS, 412, 1913

Keating, G. K., Marrone, D. P., Bower, G. C., et al. 2016, ApJ, 830, 34

Keres, D., Yun, M. S., \& Young, J. S. 2003, ApJ, 582, 659

Lagos, C. D. P., Baugh, C. M., Lacey, C. G., et al. 2011, MNRAS, 418, 1649

Lagos, C. d. P., Bayet, E., Baugh, C. M., et al. 2012, MNRAS, 426, 2142

Lagos, C. d. P., Crain, R. A., Schaye, J., et al. 2015, MNRAS, 452, 3815

Madau, P., \& Dickinson, M. 2014, ARA\&A, 52, 415

Magnelli, B., Popesso, P., Berta, S., et al. 2013, A\&A, 553, A132

Moster, B. P., Somerville, R. S., Newman, J. A., \& Rix, H.-W. 2011, ApJ, 731,113

Obreschkow, D., Heywood, I., Klöckner, H.-R., \& Rawlings, S. 2009, ApJ, 702, 1321

Pavesi, R., Sharon, C. E., Riechers, D. A., et al. 2018, ApJ, 864, 49

Popping, G., Pérez-Beaupuits, J. P., Spaans, M., Trager, S. C., \& Somerville, R. S. 2014a, MNRAS, 444, 1301
Popping, G., Somerville, R. S., \& Trager, S. C. 2014b, MNRAS, 442, 2398

Popping, G., van Kampen, E., Decarli, R., et al. 2016, MNRAS, 461, 93

Riechers, D. A., Capak, P. L., Carilli, C. L., et al. 2010, ApJL, 720, L131

Riechers, D. A., Carilli, C. L., Capak, P. L., et al. 2014, ApJ, 796, 84

Riechers, D. A., Carilli, L. C., Walter, F., et al. 2011a, ApJL, 733, L11

Riechers, D. A., Hodge, J., Walter, F., Carilli, C. L., \& Bertoldi, F. 2011b, ApJL, 739, L31

Riechers, D. A., Walter, F., Carilli, C. L., et al. 2006, ApJ, 650, 604

Saintonge, A., Catinella, B., Tacconi, L. J., et al. 2017, ApJS, 233, 22

Sargent, M. T., Béthermin, M., Daddi, E., \& Elbaz, D. 2012, ApJL, 747, L31

Sargent, M. T., Daddi, E., Béthermin, M., et al. 2014, ApJ, 793, 19

Scoville, N., Lee, N., Vanden Bout, P., et al. 2017, ApJ, 837, 150

Scoville, N., Sheth, K., Aussel, H., et al. 2016, ApJ, 820, 83

Selina, R. J., Murphy, E. J., McKinnon, M., et al. 2018, Proc. SPIE, 10700, 1070010

Sharon, C. E., Riechers, D. A., Hodge, J., et al. 2016, ApJ, 827, 18

Somerville, R. S., \& Davé, R. 2015, ARA\&A, 53, 51

Tacconi, L. J., Genzel, R., Saintonge, A., et al. 2018, ApJ, 853, 179

Tacconi, L. J., Neri, R., Genzel, R., et al. 2013, ApJ, 768, 74

Vallini, L., Gruppioni, C., Pozzi, F., Vignali, C., \& Zamorani, G. 2016, MNRAS, 456, L40

Walter, F., Decarli, R., Aravena, M., et al. 2016, ApJ, 833, 67

Walter, F., Decarli, R., Carilli, C., et al. 2012, Natur, 486, 233

Walter, F., Decarli, R., Sargent, M., et al. 2014, ApJ, 782, 79

Weyant, A., Schafer, C., \& Wood-Vasey, W. M. 2013, ApJ, 764, 116

Xie, L., De Lucia, G., Hirschmann, M., Fontanot, F., \& Zoldan, A. 2017, MNRAS, 469, 968 